\title{
A NULL CONTROLLABILITY DATA ASSIMILATION METHODOLOGY APPLIED TO A LARGE SCALE OCEAN CIRCULATION MODEL*
}

\author{
Galina C. García ${ }^{1}$, Axel Osses $^{2}$ and Jean Pierre Puel ${ }^{3}$
}

\begin{abstract}
Data assimilation refers to any methodology that uses partial observational data and the dynamics of a system for estimating the model state or its parameters. We consider here a non classical approach to data assimilation based in null controllability introduced in [Puel, C. R. Math. Acad. Sci. Paris 335 (2002) 161-166] and [Puel, SIAM J. Control Optim. 48 (2009) 1089-1111] and we apply it to oceanography. More precisely, we are interested in developing this methodology to recover the unknown final state value (state value at the end of the measurement period) in a quasi-geostrophic ocean model from satellite altimeter data, which allows in fact to make better predictions of the ocean circulation. The main idea of the method is to solve several null controllability problems for the adjoint system in order to obtain projections of the final state on a reduced basis. Theoretically, we have to prove the well posedness of the involved systems associated to the method and we also need an observability property to show the existence of null controls for the adjoint system. To this aim, we use a global Carleman inequality for the associated velocity-pressure formulation of the problem which was previously proved in [Fernández-Cara et al., J. Math. Pures Appl. 83 (2004) 1501-1542]. We present numerical simulations using a regularized version of this data assimilation methodology based on null controllability for elements of a reduced spectral basis. After proving the convergence of the regularized solutions, we analyze the incidence of the observatory size and noisy data in the recovery of the initial value for a quality prediction.
\end{abstract}

Mathematics Subject Classification. 35B37, 49J20, 35Q30, 93B05.

Received August 7, 2009. Revised April 19, 2010.

Published online August 20, 2010.

Keywords and phrases. Data assimilation, Carleman inequalities, null controllability, ocean model.

* This work was carried out under support of Stic-Amsud, MathAmsud-CIPPDE Conicyt grants and Inter-American Institute for Global Change Research (IAI) CRN II 2017 which is supported by the US National Science Foundation (Grant GEO0452325). The first and second authors acknowledge FONDECYT-Conicyt grants 1080244 and 1061263 respectively. The third authors work was partially supported by ANR C-QUID project BLAN-3-139579.

1 Departamento de Matemática y Ciencia de la Computación, Universidad de Santiago, Casilla 307, Correo 2, Santiago, Chile. galina.garcia@usach.cl

2 Departamento de Ingeniería Matemática, Universidad de Chile, Casilla 170/3 Correo 3, Santiago and Centro de Modelamiento Matemático (UMI 2807 CNRS), FCFM Universidad de Chile, Santiago, Chile. axosses@dim.uchile.cl

3 Laboratoire de Mathématiques de Versailles, Université de Versailles Saint-Quentin-en-Yvelines, 78035 Versailles, France. jppuel@math.uvsq.fr 


\section{INTRODUCTION}

A dynamical system model to approximate a physical system consists of a set of equations for each state variable of interest. In addition, we need the values of physical parameters (for example, coefficients of viscosity, diffusivity, density, etc.), forcing terms and initial and boundary conditions. In principle these values could be estimated directly from measurements. In practice, directly measuring the parameters of an ocean system is difficult because of sampling, technical and resource requirements.

The aim of data assimilation is to incorporate measured observations into a dynamical system model in order to derive accurate estimates of the current and future states of the system.

Data assimilation has been extensively used in meteorology for operational weather forecasting. On the other hand, the application of data assimilation to ocean models is more recent. For a review of the status of the subject we refer to $[2,14]$ (see also $[1,5,6,30,31]$ ).

One of the methodologies in use today is the variational method, based on optimal control theory, see for instance the works by Lions [18] and Marchuk [23] for general presentations. The idea of variational data assimilation is the following: we know "measurements" of the state on a time interval $\left(0, T_{0}\right), T_{0}>0$, and we look for the initial value at $t=0$, in order to compute the state on the time interval $\left(0, T_{0}+T\right)$. Variational data assimilation methods use optimal control theory to minimize a suitable cost function (usually weighted least square methods). This problem is known to be ill-posed but this can be partially circumvented by adding regularization terms (Tikhonov or another regularization, see $[6,8,17,22,25,26]$ ).

In [27], the author introduced an approach where we do not look for the value at $t=0$ in order to predict the evolution of the system in $\left(0, T_{0}+T\right)$. Instead, we look for the value at $t=T_{0}$, without need of the initial data at $t=0$. The idea is to compute an approximation of the state during a period of time $\left(T_{0}, T_{0}+T\right)$ using measurements of the state in some space region during a time interval $\left(0, T_{0}\right)$.

The purpose of this work is to apply this method to a simplified ocean model. Theoretically, we obtain an exact reconstruction of the state value at $t=T_{0}$ by solving null controllability problems for the adjoint system. We have to justify the existence of such exact controls and precisely derive the recovery theorem. In order to do this properly, we have to analyze the well posedness of the involved systems associated to the method and we need to prove a suitable observability property for the quasi-geostrophic system. To this aim, we use a global Carleman inequality for the corresponding velocity - pressure formulation introduced in [11]. Finally, as we use a regularization by a penalty method in order to make numerical simulations, we have to prove the convergence of the corresponding approximate recovery formula as the penalization parameter tends to zero.

The paper is organized as follows. In Section 1, we introduce the quasi-geostrophic ocean model we are considering and the data assimilation problem. We first prove, in Section 2.1, an observability inequality which is based on a global Carleman inequality for the corresponding velocity-pressure formulation of the system (2.1). In Section 2.2, we prove Theorem 1.7 which allows us to obtain an exact reconstruction of the final state. In Section 3, we give an approximate algorithm which uses a penalty method and classical optimal control auxiliary problems and we prove its convergence. Finally, in Section 4 we implement this method and present several numerical experiments using a reduced spectral basis approach. We analyze the incidence of the observatory size and noisy data in the recovery of the final state for a quality prediction. We end in Section 5 with some comments and conclusions.

\section{The OCEAN MODEL AND THE DATA ASSIMILATION PROBLEM}

Let $\Omega$ be a nonempty open bounded and simply-connected subset of $\mathbb{R}^{2}$, with boundary $\Gamma$ of class $\mathrm{C}^{2}$, and outwards unit normal vector $\nu=\nu(x)$. Given $T>0$, let us consider the linear quasi-geostrophic ocean model 
(see [3,24]) described by the following equations:

$$
\left\{\begin{array}{l}
\frac{\partial u}{\partial t}-A_{H} \Delta u+\gamma u+\left(f_{0}+\beta x_{2}\right) \mathrm{k} \wedge u+\frac{1}{\rho_{0}} \nabla p=\mathcal{T} \quad \text { in } \Omega \times(0, T), \\
\operatorname{div} u=0 \text { in } \Omega \times(0, T), \\
u=0 \text { on } \Gamma \times(0, T), \\
u(0)=u_{0} \text { in } \Omega,
\end{array}\right.
$$

where $u(x, t)$ and $p(x, t)$, respectively, denote the velocity and the pressure of the fluid at $(x, t)=\left(x_{1}, x_{2}, t\right) \in$ $\mathbb{R}^{2} \times \mathbb{R}_{+}$. In this model, $A_{H}$ represents the horizontal eddy viscosity coefficient, $\gamma$ is the bottom friction coefficient, $\rho_{0}$ is the fluid density, $\mathcal{T}$ is the wind stress, and $\left(f_{0}+\beta x_{2}\right) \mathrm{k} \wedge u$ is the Coriolis term, with $\mathrm{k} \wedge u=\left(-u_{2}, u_{1}\right)$. We have used the $\beta$-plane approximation, with $\beta=2 \Omega_{0} R^{-1} \cos \tilde{\theta}_{0}$, where $\Omega_{0}$ and $R$ are the angular velocity and radius of the Earth, respectively, and $\tilde{\theta}_{0}$ a reference latitude.

In this paper, we will consider the above ocean model formulated in terms of the stream function $\psi(x, t)$. Since $\operatorname{div} u=0, u=0$ on $\Gamma \times(0, T)$, and $\Omega$ is a connected subset of $\mathbb{R}$, we can introduce the stream function $\psi(x, t)$ satisfying:

and the following equations:

$$
u(x, t)=\operatorname{curl} \psi(x, t) \equiv\left(\frac{\partial \psi}{\partial x_{2}},-\frac{\partial \psi}{\partial x_{1}}\right),
$$

$$
\left\{\begin{array}{l}
R_{o} \frac{\partial}{\partial t}(\Delta \psi)-\epsilon_{m} \Delta^{2} \psi+\epsilon_{s} \Delta \psi+\frac{\partial \psi}{\partial x_{1}}=-\operatorname{curl} \mathcal{T} \quad \text { in } \Omega \times(0, T), \\
\psi=\frac{\partial \psi}{\partial n}=0 \quad \text { on } \Gamma \times(0, T), \\
\Delta \psi(0)=-\operatorname{curl} u_{0}=\Delta \psi_{0} \quad \text { in } \Omega
\end{array}\right.
$$

where the coefficients $R_{o}, \epsilon_{s}$ and $\epsilon_{m}$ are the non-dimensional Rossby, Stommel and Munk numbers, respectively:

$$
R_{o}=\frac{U}{\beta \mathrm{L}^{2}}, \quad \epsilon_{m}=\frac{A_{H}}{\beta L^{3}}, \quad \epsilon_{s}=\frac{\gamma}{\beta L} .
$$

In (1.2), $U$ denotes a typical horizontal velocity, $L$ is a representative horizontal length scale of ocean circulation (see $[3,24]$ for typical values).

Let $T$ be a real positive number. For any Banach space $Z$, we denote by $\mathrm{L}^{2}(Z)$ and $\mathrm{H}^{1}(Z)$ the spaces $\mathrm{L}^{2}(0, T ; Z)$ and $\mathrm{H}^{1}(0, T ; Z)$, respectively. We also consider the space $\mathrm{C}^{0}(Z)$ of continuous functions from $[0, T]$ into $Z$.

We can easily prove, by adapting the arguments of [4] to the presence of a skew-symmetric Coriolis term in the equations, the following existence result:

Proposition 1.1. For a given $\psi_{0} \in \mathrm{H}_{0}^{1}(\Omega)$ and $\mathcal{T} \in \mathrm{L}^{2}\left(\mathrm{H}^{-1}(\Omega)\right)^{2}$, problem (1.1) has a unique solution $\psi$, with $\psi \in \mathrm{L}^{2}\left(\mathrm{H}_{0}^{2}(\Omega)\right) \cap \mathrm{C}^{0}\left(\mathrm{H}_{0}^{1}(\Omega)\right)$ and $\Delta \psi \in \mathrm{H}^{1}\left(\mathrm{H}^{-2}(\Omega)\right)$. Moreover, there exists a positive constant $C$ such that

$$
\|\psi\|_{\mathrm{L}^{2}\left(\mathrm{H}_{0}^{2}(\Omega)\right) \cap \mathrm{C}^{0}\left(\mathrm{H}_{0}^{1}(\Omega)\right)}+\left\|\frac{\partial}{\partial t}(\Delta \psi)\right\|_{\mathrm{L}^{2}\left(\mathrm{H}^{-2}(\Omega)\right)} \leq C\left\{\|\mathcal{T}\|_{\mathrm{L}^{2}\left(\mathrm{H}^{-1}(\Omega)\right)^{2}}+\left\|\psi_{0}\right\|_{1, \Omega}\right\} .
$$

In fact, we can improve the regularity of the solution obtained in Proposition 1.1 (see [4]).

Proposition 1.2. For a given $\psi_{0} \in \mathrm{H}_{0}^{2}(\Omega)$ and $\mathcal{T} \in \mathrm{L}^{2}\left(\mathrm{~L}^{2}(\Omega)\right)^{2}$, problem (1.1) has a unique solution $\psi \in$ $\mathrm{L}^{2}\left(\mathrm{H}^{3}(\Omega) \cap \mathrm{H}_{0}^{2}(\Omega)\right) \cap \mathrm{C}^{0}\left(\mathrm{H}_{0}^{2}(\Omega)\right)$ and $\frac{\partial \psi}{\partial t} \in \mathrm{L}^{2}\left(\mathrm{H}_{0}^{1}(\Omega)\right)$. 
Corollary 1.3. For any $\psi_{0} \in \mathrm{H}_{0}^{1}(\Omega), \mathcal{T} \in \mathrm{L}^{2}\left(\mathrm{~L}^{2}(\Omega)\right)^{2}$, and $\delta>0$, the solution of (1.1) satisfies $\psi \in$ $\mathrm{C}^{0}\left([\delta, T], \mathrm{H}_{0}^{2}(\Omega)\right)$.

To obtain the last result, we introduce $\psi_{\delta}(x, t)=\gamma_{\delta}(t) \psi(x, t)$, where $\gamma_{\delta}$ is a regular function satisfying: $\gamma_{\delta}(0)=0,0 \leq \gamma_{\delta}(t) \leq 1$ in $(0, \delta)$, and $\gamma_{\delta}(t)=1$ in $(\delta, T)$, where $\delta$ is small enough. Now, we write the corresponding problem (1.1) for $\psi_{\delta}$, using the fact that $\psi_{\delta}(0)=0$ and Proposition 1.2, we can deduce that $\psi \in \mathrm{C}^{0}\left([\delta, T], \mathrm{H}_{0}^{2}(\Omega)\right)$, for all $\delta>0$.

An application of data assimilation in oceanography is the insertion of the altimetry satellite data into the ocean models in order to recover streamlines. In the framework of quasi-geostrophy, the sea-surface height or dynamical topography is proportional to the stream function $\left(\psi_{\text {obs }}\right)$ :

$$
\text { sea- } \text { surface height }=\frac{f_{0}}{g} \psi_{\mathrm{obs}},
$$

where $f_{0}$ is the Coriolis parameter evaluated at reference latitude $\tilde{\theta}_{0}$ and $g$ is the gravitational acceleration (see $[6,22])$.

Let us consider ocean model (1.1) where we do not impose any initial condition on $\psi$. We assume that we know the stream function $\psi_{\text {obs }}$ in a time interval $\left(0, T_{0}\right)$, with $0<T_{0}<T$, and distributed in the observation region $\mathcal{O}$, which is a non-empty open subset of $\Omega$, i.e.,

$$
\psi_{\mathrm{obs}}=\left.\psi\right|_{\mathcal{O} \times\left(0, T_{0}\right)} .
$$

Our aim is to reconstruct the value of the state at time $T_{0}$, i.e., $\psi\left(T_{0}\right)$. This value will be the initial condition for the interval $\left(T_{0}, T\right)$, where we want to predict the circulation of the ocean. From [27], the data assimilation problem consists of determining an approximation of the state value at time $T_{0}$ from the known "measurements" of the state in $\mathcal{O} \times\left(0, T_{0}\right)$.

For the reconstruction of $\psi\left(T_{0}\right)$ we will introduce a control problem for the following backward adjoint system: For $\varphi_{0}$ in an adequate space $\left(\mathrm{L}^{2}(\Omega)\right.$ or $\left.\mathrm{H}_{0}^{1}(\Omega)\right)$ and $h$ in $\mathrm{L}^{2}\left(\mathrm{~L}^{2}(\mathcal{O})\right)$, let us consider the following equation:

$$
\left\{\begin{array}{l}
-R_{o} \frac{\partial}{\partial t}(\Delta z)-\epsilon_{m} \Delta^{2} z+\epsilon_{s} \Delta z-\frac{\partial z}{\partial x_{1}}=-h 1_{\mathcal{O}} \text { in } \Omega \times\left(0, T_{0}\right), \\
z=\frac{\partial z}{\partial n}=0 \quad \text { on } \Gamma \times\left(0, T_{0}\right) \\
z\left(T_{0}\right)=\varphi_{0} \quad \text { in } \Omega
\end{array}\right.
$$

For the existence of a solution of (1.4), we will use the transposition method introduced in [21].

Definition 1.4. For each $\varphi_{0} \in \mathrm{L}^{2}(\Omega)$ and $h \in \mathrm{L}^{2}\left(\mathrm{~L}^{2}(\mathcal{O})\right)$, we say that $\left(z, z_{0}\right)$ is a weak solution of (1.4) if $z \in \mathrm{L}^{2}\left(\mathrm{H}_{0}^{1}(\Omega)\right), z_{0} \in \mathrm{H}_{0}^{1}(\Omega)$ and

$$
\int_{0}^{T_{0}}\langle f, z\rangle \mathrm{d} t-R_{o} \int_{\Omega} \nabla \theta_{0} \cdot \nabla z_{0} \mathrm{~d} x=-\int_{0}^{T_{0}} \int_{\mathcal{O}} h \theta \mathrm{d} x \mathrm{~d} t+R_{o} \int_{\Omega} \varphi_{0} \Delta \theta\left(T_{0}\right) \mathrm{d} x,
$$

for every $f \in \mathrm{L}^{2}\left(\mathrm{H}^{-1}(\Omega)\right)$ and $\theta_{0} \in \mathrm{H}_{0}^{1}(\Omega)$, where $\theta$ is the solution of

$$
\left\{\begin{array}{l}
R_{o} \frac{\partial}{\partial t}(\Delta \theta)-\epsilon_{m} \Delta^{2} \theta+\epsilon_{s} \Delta \theta+\frac{\partial \theta}{\partial x_{1}}=f \text { in } \Omega \times\left(0, T_{0}\right), \\
\theta=\frac{\partial \theta}{\partial n}=0 \quad \text { on } \Gamma \times\left(0, T_{0}\right), \\
\theta(0)=\theta_{0} \quad \text { in } \Omega .
\end{array}\right.
$$


Here, $\theta$ satisfies the same regularity as in $(1.1)$ and $\langle\cdot, \cdot\rangle$ denotes the duality pairing between $\mathrm{H}^{-1}(\Omega)$ and $\mathrm{H}_{0}^{1}(\Omega)$.

Theorem 1.5. For every $\varphi_{0} \in \mathrm{L}^{2}(\Omega)$ and $h \in \mathrm{L}^{2}\left(\mathrm{~L}^{2}(\mathcal{O})\right)$, there exists a unique weak solution $\left(z, z_{0}\right)$ in the sense of Definition 1.4 in $\mathrm{L}^{2}\left(\mathrm{H}_{0}^{1}(\Omega)\right) \times \mathrm{H}_{0}^{1}(\Omega)$. Moreover, $\Delta z \in \mathrm{C}^{0}\left(\mathrm{H}^{-2}(\Omega)\right)$ and $\Delta z(0)=\Delta z_{0}$.

Proof. Given $\varphi_{0} \in \mathrm{L}^{2}(\Omega)$ and $h \in \mathrm{L}^{2}\left(\mathrm{~L}^{2}(\mathcal{O})\right)$, let us define the functional $l: \mathrm{L}^{2}\left(\mathrm{H}^{-1}(\Omega)\right) \times \mathrm{H}_{0}^{1}(\Omega) \rightarrow \mathbb{R}$ by

$$
l\left(f, \theta_{0}\right):=-\int_{0}^{T_{0}} \int_{\mathcal{O}} h \theta \mathrm{d} x \mathrm{~d} t+R_{o} \int_{\Omega} \varphi_{0} \Delta \theta\left(T_{0}\right) \mathrm{d} x,
$$

where $\theta$ is the solution of (1.6).

Since $\theta$ satisfies Proposition 1.1, it follows that $l(\cdot, \cdot)$ is well-defined, and it is easy to prove that it defines a linear continuous functional on $\mathrm{L}^{2}\left(\mathrm{H}^{-1}(\Omega)\right) \times \mathrm{H}_{0}^{1}(\Omega)$.

From Riesz Theorem, there exists a unique pair $\left(z, z_{0}\right)$ in $\mathrm{L}^{2}\left(\mathrm{H}_{0}^{1}(\Omega)\right) \times \mathrm{H}_{0}^{1}(\Omega)$ such that

$$
l\left(f, \theta_{0}\right)=\int_{0}^{T_{0}}\langle f, z\rangle \mathrm{d} t-R_{o} \int_{\Omega} \nabla \theta_{0} \cdot \nabla z_{0} \mathrm{~d} x \quad \forall f \in \mathrm{L}^{2}\left(\mathrm{H}^{-1}(\Omega)\right), \forall \theta_{0} \in \mathrm{H}_{0}^{1}(\Omega)
$$

and $\left(z, z_{0}\right)$ satisfies

$$
\left\|\left(z, z_{0}\right)\right\|_{\mathrm{L}^{2}\left(\mathrm{H}_{0}^{1}(\Omega)\right) \times \mathrm{H}_{0}^{1}(\Omega)} \leq\|h\|_{\mathrm{L}^{2}\left(\mathrm{~L}^{2}(\mathcal{O})\right)}+\left\|\varphi_{0}\right\|_{0, \Omega} .
$$

Therefore, $\left(z, z_{0}\right)$ is a weak solution of (1.4) in the sense of the above definition.

Let us now verify that $\left(z, z_{0}\right)$ satisfy the equations in (1.4). Indeed, by taking $\theta \in \mathcal{D}(\mathcal{D}(\Omega))$ in (1.6) and (1.5), we obtain that the weak solution $z$ satisfies the first equation in (1.4) in the sense of distribution. Moreover, since $z$ satisfies the first equation in (1.4), with $z \in \mathrm{L}^{2}\left(\mathrm{H}_{0}^{1}(\Omega)\right)$, we can deduce that $\frac{\partial}{\partial t}(\Delta z) \in \mathrm{L}^{2}\left(\mathrm{H}^{-3}(\Omega)\right)$ that allows us to conclude $\Delta z \in \mathrm{C}^{0}\left(\mathrm{H}^{-2}(\Omega)\right)$.

It remains to obtain (in a weak form) the boundary condition $\frac{\partial z}{\partial n}=0$ on $\Gamma \times\left(0, T_{0}\right)$ and the "initial" condition $z\left(T_{0}\right)=\varphi_{0}$ in $\Omega$ which will be in fact $\Delta z\left(T_{0}\right)=\Delta \varphi_{0}$. To this end, we consider, in the first equation in (1.4), the test function $\theta \in \mathcal{D}\left(\mathrm{H}^{3}(\Omega) \cap \mathrm{H}_{0}^{2}(\Omega)\right)$ solution of (1.6). We integrate by parts with respect to the time and the space variables, and after comparing with (1.5) we deduce the boundary condition. The initial condition can be deduced in a similar way.

We have proved that the solution of problem (1.5)-(1.6) satisfies (1.4). Conversely, if we multiply (1.4) by the solution of (1.6), after integration by parts, we obtain (1.5), and the equivalence of both problems.

Remark 1.6. For any $\varphi_{0} \in \mathrm{L}^{2}(\Omega), h \in \mathrm{L}^{2}\left(\mathrm{~L}^{2}(\mathcal{O})\right)$, and $\delta>0$, small enough, the solution of (1.4) satisfies $z \in \mathrm{C}^{0}\left(\left[0, T_{0}-\delta\right] ; \mathrm{H}_{0}^{1}(\Omega)\right)$. This can be obtained easily studying the regularity of $z_{\delta}=\gamma_{\delta} z$, where $\gamma_{\delta}$ is a regular function such that $\gamma_{\delta}(t)=1$ in $\left(0, T_{0}-\delta\right), 0 \leq \gamma_{\delta}(t) \leq 1$ in $\left(T_{0}-\delta, T_{0}\right)$, and $\gamma_{\delta}\left(T_{0}\right)=0$. Moreover, $z_{0}=z_{\delta}(0)=z(0)$.

Now, we present the main result which gives us an exact reconstruction of $\Delta \psi\left(T_{0}\right)$.

Theorem 1.7. For any non empty $\mathcal{O} \subset \Omega, T_{0}>0$ and $\varphi_{0} \in \mathrm{L}^{2}(\Omega)$, there exists $h=h\left(\varphi_{0}\right)$ in $\mathrm{L}^{2}\left(\mathrm{~L}^{2}(\mathcal{O})\right)$ such that the solution $z$ of problem (1.4)-(1.5) satisfies

$$
z(0)=0 \quad \text { in } \Omega .
$$

We then have, for any unknown $\psi_{0} \in \mathrm{H}_{0}^{1}(\Omega)$, for any given $\mathcal{T} \in \mathrm{L}^{2}\left(\mathrm{~L}^{2}(\Omega)\right)^{2}$,

$$
\forall \varphi_{0} \in \mathrm{L}^{2}(\Omega), \quad\left(\Delta \psi\left(T_{0}\right), \varphi_{0}\right)=\frac{1}{R_{o}}\left\{\int_{0}^{T_{0}} \int_{\mathcal{O}} \psi_{\text {obs }} h\left(\varphi_{0}\right) \mathrm{d} x \mathrm{~d} t+\int_{0}^{T_{0}} \int_{\Omega} \mathcal{T} \cdot \operatorname{curl} z\left(\varphi_{0}\right) \mathrm{d} x \mathrm{~d} t\right\} .
$$


Moreover, there exists a positive constant $C$ depending on $\Omega, \mathcal{O}$ and $T_{0}$ such that

$$
\left\|\Delta \psi\left(T_{0}\right)\right\|_{0, \Omega}^{2} \leq C\left\{\int_{0}^{T_{0}} \int_{\mathcal{O}}\left|\psi_{\text {obs }}\right|^{2} \mathrm{~d} x \mathrm{~d} t+\int_{0}^{T_{0}} \int_{\Omega}|\mathcal{T}|^{2} \mathrm{~d} x \mathrm{~d} t\right\} .
$$

Equation (1.10) allows us to calculate the component of the final state $\Delta \psi\left(T_{0}\right)$ on $\varphi_{0}$ for any $\varphi_{0} \in \mathrm{L}^{2}(\Omega)$ from the wind stress $\mathcal{T} \in \mathrm{L}^{2}\left(\mathrm{~L}^{2}(\Omega)\right)^{2}$, the measurement $\psi_{\text {obs }}$, and the control $h\left(\varphi_{0}\right)$, which has to be computed. Taking successively for $\varphi_{0}$ elements of a Hilbert basis of $\mathrm{L}^{2}(\Omega)$, we can therefore reconstruct exactly $\Delta \psi\left(T_{0}\right)$. Notice that, we look for the value at $T_{0}$, without need of initial data $\psi(0)=\psi_{0}$. Inequality (1.11) can be viewed as a stability inequality.

Remark 1.8. Theorem 1.7 can be proved under the following assumptions: $\psi_{0} \in \mathrm{H}_{0}^{1}(\Omega)$ unknown, $\mathcal{T} \in$ $\mathrm{L}^{2}\left(\mathrm{H}^{-1}(\Omega)\right)^{2}$ given and for $\varphi_{0} \in \mathrm{H}_{0}^{1}(\Omega)$ and the associated solution $z$ to (1.4) satisfying Proposition 1.1. In this case we will obtain an estimate for $\left\|\Delta \psi\left(T_{0}\right)\right\|_{-1, \Omega}$.

The proof of Theorem 1.7 is reduced to studying the null controllability of system (1.4). Inequality (1.11) will be obtained from an observability inequality which will be proved in the next section.

\section{EXACT RECONSTRUCtion OF THE FinAL STATE}

The goal of this section is to prove Theorem 1.7. This proof is based on the following observability result.

\subsection{Proof of an observability inequality}

To simplify the notation, let us set $R_{o}=1, \epsilon_{m}=1$ and $\epsilon_{s}=1$.

Let $\phi$ be the solution of the following problem:

$$
\begin{cases}\frac{\partial}{\partial t}(\Delta \phi)-\Delta^{2} \phi+\Delta \phi+\frac{\partial \phi}{\partial x_{1}}=0 & \text { in } \Omega \times\left(0, T_{0}\right), \\ \phi=\frac{\partial \phi}{\partial n}=0 & \text { on } \Gamma \times\left(0, T_{0}\right), \\ \phi(0)=\phi_{0} & \text { in } \Omega\end{cases}
$$

where $\phi$ has the same regularity as in (1.1). We have the following observability inequality:

Theorem 2.1. There exists a positive constant $C$, depending only on $\Omega, \mathcal{O}$ and $T_{0}$, such that for any solution $\phi$ of (2.1), with $\phi_{0} \in \mathrm{H}_{0}^{1}(\Omega)$, we have

$$
\left\|\Delta \phi\left(T_{0}\right)\right\|_{0, \Omega}^{2} \leq C \int_{0}^{T_{0}} \int_{\mathcal{O}}|\phi|^{2} \mathrm{~d} x \mathrm{~d} t .
$$

The proof of Theorem 2.1 is based on a global Carleman inequality for a problem which is equivalent to (2.1) in terms of the original variables: the velocity $v(x, t)$ and the pressure $p(x, t)$ (see Prop. 2.4 below). For this problem, we will apply a global Carleman estimate given in $[10,11]$ and, after some computation, we arrive at the observability inequality.

Remark 2.2. For any solution $\phi$ of (2.1), with $\phi_{0} \in \mathrm{L}^{2}(\Omega)$, the observability inequality (2.1) is also valid.

Remark 2.3. In the case where $\phi=0$ and $\Delta \phi=0$ on $\Gamma \times\left(0, T_{0}\right)$, we can directly obtain a global Carleman estimate for (2.1) (see [12]). 
Proposition 2.4. Problem (2.1) with initial condition $\phi_{0} \in \mathrm{H}_{0}^{1}(\Omega)$ and $\Delta \phi_{0}=\operatorname{curl} v_{0}$, where $v_{0} \in H$, is equivalent to the following problem:

$$
\left\{\begin{array}{l}
\frac{\partial v}{\partial t}-\Delta v+v+x_{2} k \wedge v+\nabla p=0 \text { in } \Omega \times\left(0, T_{0}\right), \\
\operatorname{div} v=0 \text { in } \Omega \times\left(0, T_{0}\right), \\
v=0 \text { on } \Gamma \times\left(0, T_{0}\right), \\
v(0)=v_{0} \text { in } \Omega .
\end{array}\right.
$$

The proof of this proposition is classical, with $v=\operatorname{curl} \phi$ (see, for example, [29]). Let us remark that problem (2.3) actually has a unique solution. Indeed, using the standard spaces $H=\left\{v \in \mathrm{L}^{2}(\Omega)^{2}: \operatorname{div} v=0\right.$ in $\Omega, v \cdot \nu=0$ on $\Gamma\}$ and $V=\left\{v \in \mathrm{H}_{0}^{1}(\Omega)^{2}: \operatorname{div} v=0\right.$ in $\left.\Omega\right\}$ defined for Stokes system (see, for example, [11]), we have the following result: Given $v_{0} \in H$, problem (2.3) has a unique solution $(v, p)$, with $v \in \mathrm{L}^{2}(V) \cap \mathrm{H}^{1}\left(V^{\prime}\right) \cap$ $\mathrm{C}^{0}\left(\mathrm{~L}^{2}(\Omega)\right)$ and $p \in W^{-1,2}\left(0, T_{0} ; L_{0}^{2}(\Omega)\right)$.

Now, we will recall the Carleman estimate for $(2.3)$ given in $[10,11]$. Let us first introduce the following weight functions

$$
\begin{array}{ll}
\alpha(x, t)=\frac{\mathrm{e}^{2 \lambda\left\|\eta_{0}\right\|_{\infty}}-\mathrm{e}^{\lambda \eta_{0}}}{t^{4}\left(T_{0}-t\right)^{4}}, & \widehat{\alpha}(t)=\min _{\bar{\Omega}} \alpha(x, t), \quad \alpha^{*}(t)=\max _{\bar{\Omega}} \alpha(x, t), \\
\varphi(x, t)=\frac{\mathrm{e}^{\lambda \eta_{0}}}{t^{4}\left(T_{0}-t\right)^{4}}, & \widehat{\varphi}(t)=\max _{\bar{\Omega}} \varphi(x, t), \quad \varphi^{*}(t)=\min _{\bar{\Omega}} \varphi(x, t),
\end{array}
$$

where $\eta_{0} \in \mathrm{C}^{2}(\bar{\Omega})$ is an auxiliary function introduced in [13] which satisfies

$$
\eta_{0}>0 \text { in } \Omega, \eta_{0}=0 \text { on } \Gamma,\left|\nabla \eta_{0}\right| \geq \gamma_{0}>0 \text { in } \bar{\Omega} \backslash \mathcal{O}_{1}
$$

where $\mathcal{O}_{1}$ is a non empty open subset of $\Omega$.

Theorem 2.5. Let $\mathcal{O}_{1}$ be a non empty open subset of $\Omega$ and let the functions $\alpha, \varphi, \widehat{\alpha}$, and $\widehat{\varphi}$ be as above. For every $\gamma_{1} \in(0,1)$, there exist positive constants $s_{1}, \lambda_{1}$, and $C_{1}$, depending on $\Omega, \mathcal{O}_{1}$, and $T_{0}$ such that

$$
\begin{aligned}
I(s, \lambda ; v): & =\int_{0}^{T_{0}} \int_{\Omega} \mathrm{e}^{-2 s \alpha}\left(\frac{1}{s \varphi}\left(\left|\frac{\partial v}{\partial t}\right|^{2}+|\Delta v|^{2}\right)+s \lambda^{2} \varphi|\nabla v|^{2}+s^{3} \lambda^{4} \varphi^{3}|v|^{2}\right) \mathrm{d} x \mathrm{~d} t \\
& \leq C_{1} \int_{0}^{T_{0}} \int_{\mathcal{O}_{1}} \mathrm{e}^{-\left(1+\gamma_{1}\right) s \widehat{\alpha}} s^{7} \lambda^{4} \widehat{\varphi}^{15 / 2}|v|^{2} \mathrm{~d} x \mathrm{~d} t
\end{aligned}
$$

for any $s>s_{1}$ and $\lambda>\lambda_{1}$ and for every solution $(v, p)$ of (2.3) associated with an initial data $v_{0} \in H$.

Proof of Theorem 2.1. We will divide the proof into two steps.

Step 1. Let us first obtain $\left\|\Delta \phi\left(T_{0}\right)\right\|_{0, \Omega}^{2} \leq C I(s, \lambda ; v)$. To this end, we introduce $\tilde{\phi}(x, t)=\gamma(t) \phi(x, t)$, where $\gamma$ is a regular function satisfying:

$$
\gamma(t)=1 \text { in }\left(3 T_{0} / 4, T_{0}\right), \quad 1 \geq \gamma(t) \geq 0 \text { in }\left(T_{0} / 4,3 T_{0} / 4\right), \quad \gamma(t)=0 \text { in }\left(0, T_{0} / 4\right) .
$$

It is easy to check that $\tilde{\phi}$ verifies

$$
\left\{\begin{array}{l}
\frac{\partial}{\partial t}(\Delta \tilde{\phi})-\Delta^{2} \tilde{\phi}+\Delta \tilde{\phi}+\frac{\partial \tilde{\phi}}{\partial x_{1}}=-\gamma^{\prime} \Delta \phi \quad \text { in } \Omega \times\left(0, T_{0}\right), \\
\tilde{\phi}=\frac{\partial \tilde{\phi}}{\partial n}=0 \quad \text { on } \Gamma \times\left(0, T_{0}\right), \\
\tilde{\phi}(0)=0 \quad \text { in } \Omega .
\end{array}\right.
$$


If we multiply (2.6) by $-\frac{\partial \tilde{\phi}}{\partial t}$ and integrate by parts with respect to the space variable, we obtain

$$
\int_{\Omega}\left|\nabla \tilde{\phi}_{t}\right|^{2} \mathrm{~d} x+\frac{1}{2} \frac{\mathrm{d}}{\mathrm{d} t} \int_{\Omega}|\Delta \tilde{\phi}|^{2} \mathrm{~d} x+\frac{1}{2} \frac{\mathrm{d}}{\mathrm{d} t} \int_{\Omega}|\nabla \tilde{\phi}|^{2} \mathrm{~d} x \leq\left\|\gamma^{\prime} \nabla \phi\right\|_{0, \Omega}\left\|\nabla \tilde{\phi}_{t}\right\|_{0, \Omega}+C\|\nabla \tilde{\phi}\|_{0, \Omega}\left\|\nabla \tilde{\phi}_{t}\right\|_{0, \Omega} .
$$

We integrate in $\left[0, T_{0}\right]$ and after some computation, we get

$$
\int_{0}^{T_{0}}\left\|\nabla \tilde{\phi}_{t}\right\|_{0, \Omega}^{2} \mathrm{~d} t+\left\|\Delta \tilde{\phi}\left(T_{0}\right)\right\|_{0, \Omega}^{2}+\left\|\nabla \tilde{\phi}\left(T_{0}\right)\right\|_{0, \Omega}^{2} \leq C \int_{T_{0} / 4}^{3 T_{0} / 4}|\phi|_{1, \Omega}^{2} \mathrm{~d} t
$$

Here, we used $\int_{0}^{T_{0}}\|\nabla \tilde{\phi}\|_{0, \Omega}^{2} \mathrm{~d} t \leq C \int_{0}^{T_{0}}\left\|\gamma^{\prime} \nabla \phi\right\|_{0, \Omega}^{2} \mathrm{~d} t$, which can be obtained multiplying (2.6) by $-\tilde{\phi}$.

On the other hand, since $v(t)=\operatorname{curl} \phi(t)$,

$$
\|v(t)\|_{0, \Omega}^{2}=\|\overrightarrow{\operatorname{curl}} \phi(t)\|_{0, \Omega}^{2}=|\phi(t)|_{1, \Omega}^{2},
$$

and following the same step as in [11], we introduce the weight functions $\alpha^{*}$ and $\varphi^{*}$, defined in (2.4), to obtain

$$
\left\|\Delta \phi\left(T_{0}\right)\right\|_{0, \Omega}^{2} \leq C \int_{T_{0} / 4}^{3 T_{0} / 4}\|v(t)\|_{0, \Omega}^{2} \mathrm{~d} t \leq C \int_{T_{0} / 4}^{3 T_{0} / 4} \mathrm{e}^{-2 s \alpha^{*}} \varphi^{* 3}\|v(t)\|_{0, \Omega}^{2} \mathrm{~d} t \leq C \int_{0}^{T_{0}} \int_{\Omega} \mathrm{e}^{-2 s \alpha} \varphi^{3}|v(t)|^{2} \mathrm{~d} x \mathrm{~d} t .
$$

Notice that the last integral is bounded by $I(s, \lambda ; v)$, allowing us to deduce

$$
\left\|\Delta \phi\left(T_{0}\right)\right\|_{0, \Omega}^{2} \leq C I(s, \lambda ; v)
$$

where $C$ depends on $\Omega, \mathcal{O}$ and $T_{0}$.

Step 2. To obtain the upper bound in (2.2), we will use the Carleman estimate (2.5), with $v=$ curl $\phi$,

$$
\begin{aligned}
I(s, \lambda ; v) & \leq C \int_{0}^{T_{0}} \int_{\mathcal{O}_{1}} \mathrm{e}^{-\left(1+\gamma_{1}\right) s \widehat{\alpha}} s^{7} \lambda^{4} \widehat{\varphi}^{15 / 2}|v|^{2} \mathrm{~d} x \mathrm{~d} t=C \int_{0}^{T_{0}} \int_{\mathcal{O}_{1}} \mathrm{e}^{-\left(1+\gamma_{1}\right) s \widehat{\alpha}} s^{7} \lambda^{4} \widehat{\varphi}^{15 / 2}|\operatorname{curl} l|^{2} \mathrm{~d} x \mathrm{~d} t \\
& \leq C \int_{0}^{T_{0}} \int_{\mathcal{O}} \mathrm{e}^{-\left(1+\gamma_{1}\right) s \widehat{\alpha}} s^{7} \lambda^{4} \widehat{\varphi}^{15 / 2} \xi|\operatorname{curl} \phi|^{2} \mathrm{~d} x \mathrm{~d} t,
\end{aligned}
$$

where in the latter, we consider a function $\xi \in \mathrm{C}_{0}^{\infty}(\mathcal{O})$, with $\mathcal{O}_{1} \subset \subset \mathcal{O}$, satisfying

$$
0 \leq \xi \leq 1, \quad \xi(x)=1 \text { in } \mathcal{O}_{1}
$$

Integrating by parts over the space variable and taking into account that $v=\overrightarrow{c u r l} \phi$, we get

$$
\begin{aligned}
I(s, \lambda ; v) & \leq C \int_{0}^{T_{0}} \int_{\mathcal{O}} \mathrm{e}^{-\left(1+\gamma_{1}\right) s \widehat{\alpha}} s^{7} \lambda^{4} \widehat{\varphi}^{15 / 2}(\overrightarrow{\operatorname{curl}} \xi \cdot \operatorname{curl} \phi \phi+\xi \operatorname{curl}(\operatorname{curl} \phi) \phi) \mathrm{d} x \mathrm{~d} t \\
& \leq C \int_{0}^{T_{0}} \int_{\mathcal{O}} \mathrm{e}^{-\left(1+\gamma_{1}\right) s \widehat{\alpha}} s^{7} \lambda^{4} \widehat{\varphi}^{15 / 2}(\overrightarrow{\operatorname{curl} \xi} \cdot v \phi+\xi(\operatorname{curl} v) \phi) \mathrm{d} x \mathrm{~d} t .
\end{aligned}
$$


Notice that $\forall a>1$ there exists $\lambda_{a}>0$ such that $\forall \lambda>\lambda_{a}$ we have $\widehat{\varphi}(t)<a \varphi^{*}(t) \leq a \varphi(t)$. So applying Young's inequality, we obtain

$$
\begin{aligned}
I(s, \lambda ; v) \leq & \frac{\delta}{2} \int_{0}^{T_{0}} \int_{\Omega} \mathrm{e}^{-2 s \alpha} s^{3} \lambda^{4} \varphi^{3}|v|^{2} \mathrm{~d} x \mathrm{~d} t+C_{\delta} \int_{0}^{T_{0}} \int_{\mathcal{O}} \mathrm{e}^{-2\left(1+\gamma_{1}\right) s \widehat{\alpha}+2 s \alpha} s^{11} \lambda^{4} \widehat{\varphi}^{15} \varphi^{-3}|\phi|^{2} \mathrm{~d} x \mathrm{~d} t \\
& +\frac{\delta}{2} \int_{0}^{T_{0}} \int_{\Omega} \mathrm{e}^{-2 s \alpha^{*}} s \lambda^{2} \varphi|\operatorname{curl} v|^{2} \mathrm{~d} x \mathrm{~d} t+C_{\delta} \int_{0}^{T_{0}} \int_{\mathcal{O}} \mathrm{e}^{-2\left(1+\gamma_{1}\right) s \hat{\alpha}+2 s \alpha^{*}} s^{13} \lambda^{6} \widehat{\varphi}^{15} \varphi^{-1}|\phi|^{2} \mathrm{~d} x \mathrm{~d} t \\
\leq & \frac{\delta}{2} \int_{0}^{T_{0}} \int_{\Omega} \mathrm{e}^{-2 s \alpha}\left(s^{3} \lambda^{4} \varphi^{3}|v|^{2}+s \lambda^{2} \varphi|\nabla v|^{2}\right) \mathrm{d} x \mathrm{~d} t \\
& +C_{\delta} \int_{0}^{T_{0}} \int_{\mathcal{O}} \mathrm{e}^{-2\left(1+\gamma_{1}\right) s \hat{\alpha}+2 s \alpha^{*}} s^{13} \lambda^{6} \widehat{\varphi}^{14}|\phi|^{2} \mathrm{~d} x \mathrm{~d} t .
\end{aligned}
$$

Arguing as in [11], we introduce $\gamma_{2}$ with $0<\gamma_{2}<2 \gamma_{1}-1$. Then $\left(1+2 \gamma_{1}-\gamma_{2}\right) / 2>1$ and, we see that $\left(1+2 \gamma_{1}-\gamma_{2}\right) \widehat{\alpha} / 2>\alpha^{*}$ for $\lambda$ sufficiently large. Consequently, it can be assumed that

$$
-2\left(1+\gamma_{1}\right) \widehat{\alpha}+2 \alpha^{*}<-\left(1+\gamma_{2}\right) \widehat{\alpha},
$$

then we can replace $\mathrm{e}^{-2\left(1+\gamma_{1}\right) s \hat{\alpha}+2 s \alpha^{*}}$ by $\mathrm{e}^{-\left(1+\gamma_{2}\right) s \widehat{\alpha}}$ in the last integral in (2.9).

By choosing $\delta$ sufficiently small, we can absorb the first two terms on the right hand side of (2.9) with $I(s, \lambda ; v)$, hence

$$
I(s, \lambda ; v) \leq C \int_{0}^{T_{0}} \int_{\mathcal{O}} \mathrm{e}^{-\left(1+\gamma_{2}\right) s \widehat{\alpha}} s^{13} \lambda^{6} \widehat{\varphi}^{14}|\phi|^{2} \mathrm{~d} x \mathrm{~d} t .
$$

Since the weight $\mathrm{e}^{-\left(1+\gamma_{2}\right) s \widehat{\alpha}} \widehat{\varphi}^{14}$ is bounded (see [11]), we have

$$
I(s, \lambda ; v) \leq C \int_{0}^{T_{0}} \int_{\mathcal{O}}|\phi|^{2} \mathrm{~d} x \mathrm{~d} t,
$$

where $C$ depends on $\Omega, \mathcal{O}$ and $T_{0}$. Combining (2.10) and (2.8), we finally obtain the observability estimate:

$$
\left\|\Delta \phi\left(T_{0}\right)\right\|_{0, \Omega}^{2} \leq C \int_{0}^{T_{0}} \int_{\mathcal{O}}|\phi|^{2} \mathrm{~d} x \mathrm{~d} t .
$$

Remark 2.6. The unique continuation property for system (2.1) can be deduced from (2.10). Indeed, using similar arguments as before, we have

$$
\int_{0}^{T_{0}} \int_{\mathcal{O}}|\phi|^{2} \mathrm{~d} x \mathrm{~d} t \geq \tilde{C} I(s, \lambda ; v) \geq \tilde{C} \int_{0}^{T_{0}} \int_{\Omega} \mathrm{e}^{-2 s \alpha^{*}} \varphi^{*}|\operatorname{curl} v|^{2} \mathrm{~d} x \mathrm{~d} t=\tilde{C} \int_{0}^{T_{0}} \int_{\Omega} \mathrm{e}^{-2 s \alpha^{*}} \varphi^{*}|\Delta \phi|^{2} \mathrm{~d} x \mathrm{~d} t .
$$

If $\phi=0$ in $\mathcal{O} \times\left(0, T_{0}\right)$ we necessarily have $\Delta \phi \equiv 0$ in $\Omega \times\left(0, T_{0}\right)$. On the other hand, $\phi$ satisfies

$$
\left\{\begin{array}{l}
\Delta \phi(t)=0 \text { in } \Omega, t \in\left(0, T_{0}\right) \\
\phi=0 \text { in } \mathcal{O} \times\left(0, T_{0}\right)
\end{array}\right.
$$

then, it follows also that $\phi \equiv 0$ in $\Omega \times\left(0, T_{0}\right)$. 


\subsection{Proof of Theorem 1.7}

The proof of Theorem 1.7 is based on the observability result proved in Theorem 2.1 and the unique continuation property given in Remark 2.6. We argue as in $[9,19]$.

First, we will prove the approximate controllability by minimizing certain functional. Given $\varphi_{0} \in \mathrm{L}^{2}(\Omega)$ and $\varepsilon>0$, we look for the control $h=h_{\varepsilon} \in \mathrm{L}^{2}\left(\mathrm{~L}^{2}(\Omega)\right)$ with minimum $\mathrm{L}^{2}\left(\mathrm{~L}^{2}(\Omega)\right)$-norm such that $\|z(0)\|_{1, \Omega} \leq \varepsilon$. More precisely, let $\hat{\phi}_{\varepsilon}$ be the solution of

$$
\begin{cases}\frac{\partial}{\partial t}\left(\Delta \hat{\phi}_{\varepsilon}\right)-\Delta^{2} \hat{\phi}_{\varepsilon}+\Delta \hat{\phi}_{\varepsilon}+\frac{\partial \hat{\phi}_{\varepsilon}}{\partial x_{1}}=0 & \text { in } \Omega \times\left(0, T_{0}\right), \\ \hat{\phi}_{\varepsilon}=\frac{\partial \phi}{\partial n}=0 & \text { on } \Gamma \times\left(0, T_{0}\right) \\ \hat{\phi}_{\varepsilon}(0)=\phi_{0 \varepsilon} & \text { in } \Omega\end{cases}
$$

where $\phi_{0 \varepsilon} \in \mathrm{H}_{0}^{1}(\Omega)$ minimizes the following functional

$$
J_{\varepsilon}\left(\phi_{0}\right)=\frac{1}{2} \int_{0}^{T_{0}} \int_{\mathcal{O}}|\phi|^{2} \mathrm{~d} x \mathrm{~d} t+\varepsilon\left\|\phi_{0}\right\|_{1, \Omega}-\left(\Delta \phi\left(T_{0}\right), \varphi_{0}\right) .
$$

If we take

$$
h_{\varepsilon}=\hat{\phi}_{\varepsilon} 1_{\mathcal{O}}
$$

then, the associated solution $z_{\varepsilon}$ to (1.4) satisfies $\left\|z_{\varepsilon}(0)\right\|_{1, \Omega} \leq \varepsilon$.

Let us verify the existence and uniqueness of the minimum of $J_{\varepsilon}$.

Lemma 2.7. For $\varepsilon>0$, the functional $J_{\varepsilon}$ defined in (2.11) is continuous, strictly convex and satisfies

$$
\liminf _{\left\|\phi_{0}\right\|_{1, \Omega} \rightarrow \infty} \frac{J_{\varepsilon}\left(\phi_{0}\right)}{\left\|\phi_{0}\right\|_{1, \Omega}} \geq \varepsilon
$$

Proof. The proof of this inequality is classical (see [9]). For completeness we include it here. $J_{\varepsilon}$ is strictly convex since it is the sum of convex and strictly convex terms. The strict convexity of the first term is not straightforward but is a consequence of the unique continuation property. To see that $J_{\varepsilon}$ is continuous, we only need to recall the continuity property of system (2.1) (Prop. 1.1, Cor. 1.3),

$$
\|\phi\|_{\mathrm{L}^{2}\left(H_{0}^{2}(\Omega)\right) \cap \mathrm{C}^{0}\left(\mathrm{H}_{0}^{1}(\Omega)\right)}+\left\|\Delta \phi\left(T_{0}\right)\right\|_{0, \Omega} \leq C\left\|\phi_{0}\right\|_{1, \Omega}
$$

To prove (2.13), we take a sequence $\left\{\phi_{0}^{n}\right\}_{n \geq 1}$, with $\left\|\phi_{0}^{n}\right\|_{1, \Omega} \rightarrow+\infty$ and we denote by $\phi^{n}$ the solution of (2.1) associated with $\phi_{0}^{n}$. If we define

$$
\tilde{\phi}^{n}=\frac{\phi^{n}}{\left\|\phi_{0}^{n}\right\|_{1, \Omega}}, \quad \text { and } \quad \tilde{\phi}_{0}^{n}=\frac{\phi_{0}^{n}}{\left\|\phi_{0}^{n}\right\|_{1, \Omega}},
$$

then from the continuity result (2.14), we deduce that $\tilde{\phi}^{n}$ is bounded in $\mathrm{L}^{2}\left(H_{0}^{2}(\Omega)\right)$. Since $\left\|\tilde{\phi}_{0}^{n}\right\|_{1, \Omega}=1, \tilde{\phi}_{0}^{n}$ is also bounded in $\mathrm{H}_{0}^{1}(\Omega)$. Then we can extract subsequences (still denoted by $\tilde{\phi}^{n}$ and $\tilde{\phi}_{0}^{n}$ ), such that

$$
\begin{aligned}
& \tilde{\phi}^{n} \rightarrow \tilde{\phi} \quad \text { weakly in } \mathrm{L}^{2}\left(\mathrm{H}_{0}^{2}(\Omega)\right), \\
& \tilde{\phi}_{0}^{n} \rightarrow \tilde{\phi}_{0} \quad \text { weakly in } \mathrm{H}_{0}^{1}(\Omega) .
\end{aligned}
$$

On the other hand,

$$
\frac{J_{\varepsilon}\left(\phi_{0}^{n}\right)}{\left\|\phi_{0}^{n}\right\|_{1, \Omega}}=\frac{1}{2}\left\|\phi_{0}^{n}\right\|_{1, \Omega} \int_{0}^{T_{0}} \int_{\mathcal{O}}\left|\tilde{\phi}^{n}\right|^{2} \mathrm{~d} x \mathrm{~d} t+\varepsilon-\left(\Delta \tilde{\phi}^{n}\left(T_{0}\right), \varphi_{0}\right) .
$$


We have two possibilities:

(i) If $\liminf \int_{n} \int_{0}^{T_{0}} \int_{\Omega}\left|\tilde{\phi}^{n}\right|^{2} \mathrm{~d} x \mathrm{~d} t>0$. Since $\left\|\phi_{0}^{n}\right\|_{1, \Omega} \rightarrow+\infty$, inequality (2.15) implies that

$$
\liminf _{n} \frac{J_{\varepsilon}\left(\phi_{0}^{n}\right)}{\left\|\phi_{0}^{n}\right\|_{1, \Omega}} \rightarrow+\infty \geq \varepsilon
$$

(ii) If $\liminf \int_{n} \int_{0}^{T_{0}} \int_{\mathcal{O}}\left|\tilde{\phi}^{n}\right|^{2} \mathrm{~d} x \mathrm{~d} t=0$. Using the continuity and convexity property of $\|\cdot\|_{\mathrm{L}^{2}\left(\mathrm{~L}^{2}(\mathcal{O})\right)}^{2}$ and the weak-convergence of $\tilde{\phi}^{n}$, we can deduce that

$$
\int_{0}^{T_{0}} \int_{\mathcal{O}}|\tilde{\phi}|^{2} \mathrm{~d} x \mathrm{~d} t \leq \liminf _{n} \int_{0}^{T_{0}} \int_{\mathcal{O}}\left|\tilde{\phi}^{n}\right|^{2} \mathrm{~d} x \mathrm{~d} t=0
$$

Then $\tilde{\phi}=0$ in $\mathcal{O} \times\left(0, T_{0}\right)$. We now use the unique continuation result that was proved in Remark 2.6 which implies that $\tilde{\phi}=0$ in $\Omega \times\left(0, T_{0}\right)$. Therefore,

$$
\Delta \tilde{\phi}^{n}\left(T_{0}\right) \rightarrow \Delta \tilde{\phi}\left(T_{0}\right)=0 \quad \text { weakly in } \mathrm{L}^{2}(\Omega) .
$$

Using this fact, from (2.15) we obtain

$$
\liminf _{n} \frac{J_{\varepsilon}\left(\phi_{0}^{n}\right)}{\left\|\phi_{0}^{n}\right\|_{1, \Omega}}=\frac{1}{2} \liminf _{n}\left\|\phi_{0}^{n}\right\|_{1, \Omega} \int_{0}^{T_{0}} \int_{\mathcal{O}}\left|\tilde{\phi}^{n}\right|^{2} \mathrm{~d} x \mathrm{~d} t+\varepsilon-0 \geq \varepsilon
$$

and we conclude that (2.13) holds.

As an immediate consequence of this lemma, we know that for every $\varepsilon>0$, the functional $J_{\varepsilon}$ has a unique minimum $\hat{\phi}_{0 \varepsilon}$. Either we have $\hat{\phi}_{0 \varepsilon}=0$ and we are in the trivial case where we take a null control, or the following optimality condition must be satisfied:

$$
\int_{0}^{T_{0}} \int_{\mathcal{O}} \hat{\phi}_{\varepsilon} \phi \mathrm{d} x \mathrm{~d} t+\varepsilon \int_{\Omega} \frac{\nabla \hat{\phi}_{0 \varepsilon}}{\left\|\hat{\phi}_{0 \varepsilon}\right\|_{1, \Omega}} \cdot \nabla \phi_{0} \mathrm{~d} x-\int_{\Omega} \Delta \phi\left(T_{0}\right) \varphi_{0} \mathrm{~d} x=0,
$$

where $\phi$ is the solution of $(2.1)$ with $\phi(0)=\phi_{0}$. If we take the control as

$$
h_{\varepsilon}=\hat{\phi}_{\varepsilon} 1_{\mathcal{O}}
$$

and the associated solution $\hat{z}_{\varepsilon}$ to (1.4)-(1.5), then multiplying (1.4) by $\phi$ and integrating by parts, we deduce from (1.5) that

$$
\int_{\Omega} \Delta \phi\left(T_{0}\right) \varphi_{0} \mathrm{~d} x+\int_{\Omega} \nabla \phi_{0} \cdot \nabla \hat{z}_{\varepsilon}(0) \mathrm{d} x=\int_{0}^{T_{0}} \int_{\mathcal{O}} h_{\varepsilon} \phi \mathrm{d} x \mathrm{~d} t .
$$

From (2.16)-(2.18), we get

$$
\int_{\Omega}\left(\nabla \hat{z}_{\varepsilon}(0)+\varepsilon \frac{\nabla \hat{\phi}_{0 \varepsilon}}{\left\|\hat{\phi}_{0 \varepsilon}\right\|_{1, \Omega}}\right) \cdot \nabla \phi_{0} \mathrm{~d} x=0 \quad \forall \phi_{0} \in \mathrm{H}_{0}^{1}(\Omega),
$$

then,

$$
\left\|\hat{z}_{\varepsilon}(0)\right\|_{1, \Omega} \leq \varepsilon .
$$

Here, we have obtained the approximate controllability result by minimizing functionals like (2.11). 
Next, we choose $\phi_{0}=\hat{\phi}_{0 \varepsilon}$ in (2.16), applying Young's inequality, and using the observability inequality (2.2), we obtain (for $a>0$ )

$$
\int_{0}^{T_{0}} \int_{\mathcal{O}}\left|\hat{\phi}_{\varepsilon}\right|^{2} \mathrm{~d} x \mathrm{~d} t+\varepsilon\left\|\hat{\phi}_{0 \varepsilon}\right\|_{0, \Omega} \leq \frac{1}{2 a^{2}}\left\|\varphi_{0}\right\|_{0, \Omega}^{2}+\frac{a^{2}}{2}\left\|\Delta \hat{\phi}_{\varepsilon}\left(T_{0}\right)\right\|_{0, \Omega}^{2} \leq \frac{1}{2 a^{2}}\left\|\varphi_{0}\right\|_{0, \Omega}^{2}+\frac{C a^{2}}{2} \int_{0}^{T_{0}} \int_{\mathcal{O}}\left|\hat{\phi}_{\varepsilon}\right|^{2} \mathrm{~d} x \mathrm{~d} t .
$$

Choosing $a^{2}=1 / C$ and taking into account that $h_{\varepsilon}=\hat{\phi}_{\varepsilon} 1_{\mathcal{O}}$, we deduce that

$$
\int_{0}^{T_{0}} \int_{\mathcal{O}}\left|h_{\varepsilon}\right|^{2} \mathrm{~d} x \mathrm{~d} t \leq C\left\|\varphi_{0}\right\|_{0, \Omega}^{2}
$$

Since $h_{\varepsilon}$ is uniformly bounded in $\mathrm{L}^{2}\left(\mathrm{~L}^{2}(\mathcal{O})\right)$, and from Remark 1.6, $\hat{z}_{\varepsilon}$ is uniformly bounded in $\mathrm{C}^{0}\left(\left[0, T_{0}-\delta\right] ; H_{0}^{1}(\Omega)\right)$, we can extract a subsequence $\left\{h_{\varepsilon_{n}}\right\}$ and $\left\{z_{\varepsilon_{n}}\right\}$, with $\varepsilon_{n} \rightarrow 0$, such that

$$
\begin{aligned}
h_{\varepsilon_{n}} & \rightarrow h \quad \text { weakly in } \mathrm{L}^{2}\left(\mathrm{~L}^{2}(\mathcal{O})\right), \\
\hat{z}_{\varepsilon_{n}} & \rightarrow z \quad \text { weakly in } \mathrm{L}^{2}\left(0, T_{0}-\delta ; H_{0}^{2}(\Omega)\right), \\
\frac{\partial \hat{z}_{\varepsilon_{n}}}{\partial t} & \rightarrow \frac{\partial z}{\partial t} \quad \text { weakly in } \mathrm{L}^{2}\left(0, T_{0}-\delta ; \mathrm{L}^{2}(\Omega)\right),
\end{aligned}
$$

then

$$
\hat{z}_{\varepsilon_{n}}(0) \rightarrow z(0) \quad \text { in } \mathrm{H}_{0}^{1}(\Omega),
$$

as $n \rightarrow+\infty$. Here, we have denoted by $z_{\varepsilon_{n}}$ and $z$ the solutions to (1.4) associated with $h_{\varepsilon_{n}}$ and $h$, respectively. From (2.20), we deduce that $z(0)=0$ in $\Omega$.

To obtain (1.10), we use (1.1) on the time interval $\left(0, T_{0}\right)$ and (1.4)-(1.5), and the fact that $z_{0}=z(0)=0$ in $\Omega$. To deduce (1.11), we use (1.8), (1.10) and (2.21) as follows:

$$
\begin{aligned}
\left(\Delta \psi\left(T_{0}\right), \varphi_{0}\right) & =\left\{\int_{0}^{T_{0}} \int_{\mathcal{O}} \psi_{\text {obs }} h\left(\varphi_{0}\right) \mathrm{d} x \mathrm{~d} t-\int_{0}^{T_{0}} \int_{\Omega} \mathcal{T} \cdot \operatorname{curl} z\left(\varphi_{0}\right) \mathrm{d} x \mathrm{~d} t\right\} \\
& \leq C\left\|\varphi_{0}\right\|_{0, \Omega}\left(\int_{0}^{T_{0}} \int_{\mathcal{O}}\left|\psi_{\text {obs }}\right|^{2} \mathrm{~d} x \mathrm{~d} t+\int_{0}^{T_{0}} \int_{\Omega}|\mathcal{T}|^{2} \mathrm{~d} x \mathrm{~d} t\right)^{1 / 2} .
\end{aligned}
$$

Then, it follows that

$$
\left\|\Delta \psi\left(T_{0}\right)\right\|_{0, \Omega}^{2} \leq C\left\{\int_{0}^{T_{0}} \int_{\mathcal{O}}\left|\psi_{\text {obs }}\right|^{2} \mathrm{~d} x \mathrm{~d} t+\int_{0}^{T_{0}} \int_{\Omega}|\mathcal{T}|^{2} \mathrm{~d} x \mathrm{~d} t\right\} .
$$

This concludes the proof of Theorem 1.7.

\section{Approximation by an optimal CONTRol Problem}

In this section we present another method to prove approximate controllability which is useful for numerical purposes. This method uses an optimal control problem. We will be able to characterize the control of minimal norm in $\mathrm{L}^{2}\left(\mathrm{~L}^{2}(\Omega)\right)$ by an optimality system and then we will present the time-space discretization of this system.

Let us consider the following optimal control problem for fixed $\varphi_{0} \in \mathrm{L}^{2}(\Omega)$. Let $z$ be the solution of (1.4) and, for $\alpha>0$, let us define

$$
J_{\alpha}(h)=\int_{0}^{T_{0}} \int_{\mathcal{O}}|h|^{2} \mathrm{~d} x \mathrm{~d} t+\frac{1}{2 \alpha}\|z(0)\|_{1, \Omega}^{2},
$$


where we have penalized the final condition (1.9). We look for $h_{\alpha} \in \mathrm{L}^{2}\left(\mathrm{~L}^{2}(\mathcal{O})\right)$ such that

$$
J_{\alpha}\left(h_{\alpha}\right)=\min _{h \in \mathrm{L}^{2}\left(\mathrm{~L}^{2}(\mathcal{O})\right)} J_{\alpha}(h) .
$$

\section{Theorem 3.1.}

(i) For every $\alpha>0$, there exists a unique solution $h_{\alpha}$ to (3.2) and $h_{\alpha}$ is characterized by the following optimality system:

$$
\begin{aligned}
& \left\{\begin{array}{l}
-\frac{\partial}{\partial t}\left(\Delta z_{\alpha}\right)-\Delta^{2} z_{\alpha}+\Delta z_{\alpha}-\frac{\partial z_{\alpha}}{\partial x_{1}}=-h_{\alpha} 1_{\mathcal{O}} \text { in } \Omega \times\left(0, T_{0}\right), \\
z_{\alpha}=\frac{\partial z_{\alpha}}{\partial n}=0 \quad \text { on } \Gamma \times\left(0, T_{0}\right), \\
z_{\alpha}\left(T_{0}\right)=\varphi_{0} \quad \text { in } \Omega,
\end{array}\right. \\
& \left\{\begin{array}{l}
\frac{\partial}{\partial t}\left(\Delta \phi_{\alpha}\right)-\Delta^{2} \phi_{\alpha}+\Delta \phi_{\alpha}+\frac{\partial \phi_{\alpha}}{\partial x_{1}}=0 \quad \text { in } \Omega \times\left(0, T_{0}\right), \\
\phi_{\alpha}=\frac{\partial \phi_{\alpha}}{\partial n}=0 \quad \text { on } \Gamma \times\left(0, T_{0}\right), \\
-\alpha \phi_{\alpha}(0)=z_{\alpha}(0) \text { in } \Omega,
\end{array}\right. \\
& h_{\alpha}=\phi_{\alpha} 1_{\mathcal{O}} \text { in } \mathcal{O} \times\left(0, T_{0}\right) .
\end{aligned}
$$

(ii) When $\alpha$ tends to zero, we have $h_{\alpha} \rightarrow h\left(\varphi_{0}\right)$ in $\mathrm{L}^{2}\left(\mathrm{~L}^{2}(\mathcal{O})\right)$ where $h\left(\varphi_{0}\right)$ is the solution of the null controllability problem given by Theorem 1.7 which minimizes the $\mathrm{L}^{2}\left(\mathrm{~L}^{2}(\mathcal{O})\right)$-norm and

$$
\left\{\int_{0}^{T_{0}} \int_{\mathcal{O}} \psi_{\text {obs }} h_{\alpha}\left(\varphi_{0}\right) \mathrm{d} x \mathrm{~d} t-\int_{0}^{T_{0}} \int_{\Omega} \mathcal{T} \cdot \operatorname{curl} z_{\alpha}\left(\varphi_{0}\right) \mathrm{d} x \mathrm{~d} t\right\} \rightarrow\left(\Delta \psi\left(T_{0}\right), \varphi_{0}\right) .
$$

Proof.

(i) It follows from [18] that problem (3.2) has a unique solution $h_{\alpha}$ which is characterized by the optimality system (3.3)-(3.4).

(ii) Combining (3.3) and (3.4) and taking into account that $h_{\alpha}=\phi_{\alpha} 1_{\mathcal{O}}$, we obtain the following optimality condition

$$
\int_{0}^{T_{0}} \int_{\mathcal{O}}\left|h_{\alpha}\right|^{2} \mathrm{~d} x \mathrm{~d} t+\frac{1}{\alpha}\left\|z_{\alpha}(0)\right\|_{1, \Omega}^{2}=\left(\Delta \phi_{\alpha}\left(T_{0}\right), \varphi_{0}\right)
$$

Applying Young's inequality in (3.7) and using the observability result (2.2) we are lead to

$$
\begin{aligned}
\int_{0}^{T_{0}} \int_{\mathcal{O}}\left|h_{\alpha}\right|^{2} \mathrm{~d} x \mathrm{~d} t+\frac{1}{\alpha}\left\|z_{\alpha}(0)\right\|_{0, \Omega}^{2} \leq \frac{a^{2}}{2}\left\|\Delta \phi_{\alpha}\left(T_{0}\right)\right\|_{0, \Omega}^{2}+\frac{1}{2 a^{2}}\left\|\varphi_{0}\right\|_{0, \Omega}^{2} & \\
& \leq \frac{C a^{2}}{2} \int_{0}^{T_{0}} \int_{\mathcal{O}}\left|\phi_{\alpha}\right|^{2} \mathrm{~d} x \mathrm{~d} t+\frac{1}{2 a^{2}}\left\|\varphi_{0}\right\|_{0, \Omega}^{2},
\end{aligned}
$$

for $a>0$. Choosing $a^{2}=1 / C$, we can deduce that

$$
\int_{0}^{T_{0}} \int_{\mathcal{O}}\left|h_{\alpha}\right|^{2} \mathrm{~d} x \mathrm{~d} t+\frac{2}{\alpha}\left\|z_{\alpha}(0)\right\|_{1, \Omega}^{2} \leq C\left\|\varphi_{0}\right\|_{1, \Omega}^{2}
$$


Since $h_{\alpha}$ is uniformly bounded in $\mathrm{L}^{2}\left(\mathrm{~L}^{2}(\mathcal{O})\right)$, and from from Remark 1.6, $z_{\alpha}$ is uniformly bounded in $\mathrm{C}^{0}\left(\left[0, T_{0}-\delta\right] ; H_{0}^{1}(\Omega)\right)$, we can extract a subsequence $\left\{h_{\alpha_{n}}\right\}$ and $\left\{z_{\alpha_{n}}\right\}$, with $\alpha_{n} \rightarrow 0$, such that

$$
\begin{aligned}
h_{\alpha_{n}} & \rightarrow h \text { weakly in } \mathrm{L}^{2}\left(\mathrm{~L}^{2}(\mathcal{O})\right), \\
z_{\alpha_{n}} & \rightarrow z \text { weakly in } \mathrm{L}^{2}\left(0, T_{0}-\delta ; \mathrm{H}_{0}^{2}(\Omega)\right) \cap \mathrm{L}^{2}\left(0, T_{0} ; \mathrm{H}_{0}^{1}(\Omega)\right), \\
\frac{\partial z_{\alpha_{n}}}{\partial t} & \rightarrow \frac{\partial z}{\partial t} \quad \text { weakly in } \mathrm{L}^{2}\left(0, T_{0}-\delta ; \mathrm{L}^{2}(\Omega)\right),
\end{aligned}
$$

then

$$
z_{\alpha_{n}}(0) \rightarrow z(0) \quad \text { in } \mathrm{H}_{0}^{1}(\Omega),
$$

as $n \rightarrow+\infty$. Here, we have denoted by $z_{\alpha_{n}}$ and $z$ the solutions of (1.4) associated with $h_{\alpha_{n}}$ and $h$ respectively. From (3.8) and (3.9), we deduce that $z(0)=0$ in $\Omega$. Hence, we conclude, for fixed $\varphi_{0}$,

$\int_{0}^{T_{0}} \int_{\mathcal{O}} h_{\alpha_{n}} \psi_{\text {obs }} \mathrm{d} x \mathrm{~d} t-\int_{0}^{T_{0}} \int_{\Omega} \mathcal{T} \cdot \operatorname{curl} z_{\alpha_{n}} \mathrm{~d} x \mathrm{~d} t \rightarrow \int_{0}^{T_{0}} \int_{\mathcal{O}} h \psi_{\text {obs }} \mathrm{d} x \mathrm{~d} t-\int_{0}^{T_{0}} \int_{\Omega} \mathcal{T} \cdot \operatorname{curl} z \mathrm{~d} x \mathrm{~d} t=\left(\Delta \psi\left(T_{0}\right), \varphi_{0}\right)$.

Hence, we have proved (3.6). It is also clear that the limit control $h$ is one solution $h\left(\varphi_{0}\right)$ given by Theorem 1.7. By standard arguments it is easy to show that this solution is the one which minimizes the $\mathrm{L}^{2}\left(\mathrm{~L}^{2}(\mathcal{O})\right)$-norm and that the convergence of $h_{\alpha}$ towards $h=h\left(\varphi_{0}\right)$ is strong in $\mathrm{L}^{2}\left(\mathrm{~L}^{2}(\mathcal{O})\right)$.

In the following analysis, we will split the solution of (3.3) into two problems. For this, let us introduce $\tilde{z}$, the solution of

$$
\left\{\begin{array}{l}
-\frac{\partial}{\partial t}(\Delta \tilde{z})-\Delta^{2} \tilde{z}+\Delta \tilde{z}-\frac{\partial \tilde{z}}{\partial x_{1}}=0 \quad \text { in } \Omega \times\left(0, T_{0}\right) \\
\tilde{z}=\frac{\partial \tilde{z}}{\partial n}=0 \quad \text { on } \Gamma \times\left(0, T_{0}\right) \\
\tilde{z}\left(T_{0}\right)=\varphi_{0} \quad \text { in } \Omega
\end{array}\right.
$$

and then

$$
z_{\alpha}=z+\tilde{z}
$$

where $z$ is the solution of

$$
\left\{\begin{array}{l}
-\frac{\partial}{\partial t}(\Delta z)-\Delta^{2} z+\Delta z-\frac{\partial z}{\partial x_{1}}=-\phi_{\alpha} 1_{\mathcal{O}} \quad \text { in } \Omega \times\left(0, T_{0}\right) \\
z=\frac{\partial z}{\partial n}=0 \quad \text { on } \Gamma \times\left(0, T_{0}\right) \\
z\left(T_{0}\right)=0 \quad \text { in } \Omega
\end{array}\right.
$$

To simplify the notation, let us denote the function $\phi_{\alpha}(0)$ by $e$ and consider the linear operator $\Lambda: \mathrm{H}_{0}^{1}(\Omega) \rightarrow$ $\mathrm{H}_{0}^{1}(\Omega)$ defined by

$$
\Lambda e=z(0)
$$

where $z$ is obtained from $e$ as follows: First we solve (3.4) with $\phi_{\alpha}(0)=e \in \mathrm{H}_{0}^{1}(\Omega)$, and then the backward system (3.11).

Using the fact that $-\alpha e=z_{\alpha}(0)=z(0)+\tilde{z}(0)$, the optimality system (3.3)-(3.5) reduces to the following:

Find $e \in \mathrm{H}_{0}^{1}(\Omega)$ such that

$$
(\alpha I+\Lambda) e=-\tilde{z}(0)
$$

where $I$ is the identity matrix. 
The operator $\Lambda \in \mathcal{L}\left(\mathrm{H}_{0}^{1}(\Omega), \mathrm{H}_{0}^{1}(\Omega)\right)$ satisfies for all $e$ and $\tilde{e}$ in $\mathrm{H}_{0}^{1}(\Omega)$ :

$$
(\Lambda e, \tilde{e})_{1, \Omega}=\int_{\Omega} \nabla z(0) \cdot \nabla \xi(0) \mathrm{d} x=\int_{0}^{T_{0}} \int_{\mathcal{O}} \phi_{\alpha} \xi \mathrm{d} x \mathrm{~d} t,
$$

where $(\cdot, \cdot)_{1, \Omega}$ denotes the scalar product of $\mathrm{H}_{0}^{1}(\Omega)$, and $\xi$ is the solution of the following problem with initial condition $\tilde{e} \in \mathrm{H}_{0}^{1}(\Omega)$ :

$$
\left\{\begin{array}{l}
\frac{\partial}{\partial t}(\Delta \xi)-\Delta^{2} \xi+\Delta \xi+\frac{\partial \xi}{\partial x_{1}}=0 \quad \text { in } \Omega \times\left(0, T_{0}\right), \\
\xi=\frac{\partial \xi}{\partial n}=0 \quad \text { on } \Gamma \times\left(0, T_{0}\right), \\
\xi(0)=\tilde{e} \quad \text { in } \Omega
\end{array}\right.
$$

Indeed, the last equation in (3.13) can be obtained from (3.11) and (3.14).

On the other hand, it follows from (3.13) that, for all $e$ and $\tilde{e}$ in $\mathrm{H}_{0}^{1}(\Omega)$,

$$
(\Lambda e, \tilde{e})_{1, \Omega}=(\Lambda \tilde{e}, e)_{1, \Omega} \text { and }(\Lambda e, e)_{1, \Omega} \geq 0,
$$

i.e., $\Lambda$ is self-adjoint and positive semi-definite. We also have, from the unique continuation property (see Rem. 2.6), that $\Lambda$ is positive definite. Indeed, from (3.13),

$$
(\Lambda e, e)_{1, \Omega}=\int_{0}^{T_{0}} \int_{\mathcal{O}}\left|\phi_{\alpha}\right|^{2} \mathrm{~d} x \mathrm{~d} t=0 \Rightarrow \phi_{\alpha}=0 \operatorname{in} \mathcal{O} \times\left(0, T_{0}\right),
$$

but we know that necessarily $\phi_{\alpha} \equiv 0$ in $\Omega \times\left(0, T_{0}\right)$ and $\phi_{\alpha}(0)=e=0$. Notice that, if $\alpha$ is strictly positive, the operator $(\alpha I+\Lambda)$ is strongly elliptic from $\mathrm{H}_{0}^{1}(\Omega)$ to $\mathrm{H}_{0}^{1}(\Omega)$, i.e.,

$$
((\alpha I+\Lambda) e, e)_{1, \Omega}=\alpha\|e\|_{1, \Omega}^{2}+(\Lambda e, e)_{1, \Omega} \geq \alpha\|e\|_{1, \Omega}^{2} .
$$

Then, equation (3.12) allows us to calculate the optimal initial condition and using this value in (3.4), we obtain the optimal control associated with $\varphi_{0}$.

In summary, given $\varphi_{0} \in \mathrm{L}^{2}(\Omega)$, in order to find an approximation to $\left(\Delta \psi\left(T_{0}\right), \varphi_{0}\right)$, we first have to solve an optimal control problem for each $\varphi_{0}$, which reduces to solving (3.12). Operator $\Lambda$ on the left-hand side of (3.12) represents the coupled optimality system (3.4) and (3.11) which does not depend on $\varphi_{0}$. The right-hand side of (3.12) depends on $\varphi_{0}$ and corresponds to solving (3.10). This is very important for the numerical approximation because, after discretization, all the linear systems corresponding to different $\varphi_{0}$ have the same matrices [27]. For $\varphi_{0}$, we would take an appropriate finite dimensional basis. Once the optimal control and states solutions of (3.12) are known, they are used with source terms and observations in formula (1.10) in order to compute the projection $\left(\Delta \psi\left(T_{0}\right), \varphi_{0}\right)$.

\subsection{Approximation of problems (3.3)-(3.4)}

In this section we present the time-space discretization of problem (3.3)-(3.4) formulated in terms of the stream function and the vorticity. Here we use a combination of time discretization by finite differences and space discretization by finite elements. The methodology used in this work to implement the data assimilation method is simple. Similar studies of the numerical solution of the approximate controllability problems are discussed in [20] for diffusion equations. 
Let us rewrite problem (3.3)-(3.4) by introducing the following variables $s_{\alpha}=-\Delta z_{\alpha}$ and $\theta_{\alpha}=-\Delta \phi_{\alpha}$. As before, we will split the solution of (3.3) into two problems. Let $(\tilde{z}, \tilde{s})$ be the solution of

$$
\left\{\begin{array}{l}
-\frac{\partial \tilde{s}}{\partial t}-\Delta \tilde{s}+\tilde{s}+\frac{\partial \tilde{z}}{\partial x_{1}}=0 \quad \text { in } \Omega \times\left(0, T_{0}\right) \\
\tilde{s}+\Delta \tilde{z}=0 \quad \text { in } \Omega \times\left(0, T_{0}\right) \\
\tilde{z}=\frac{\partial \tilde{z}}{\partial n}=0 \quad \text { on } \Gamma \times\left(0, T_{0}\right) \\
\tilde{z}\left(T_{0}\right)=\varphi_{0} \quad \text { in } \Omega
\end{array}\right.
$$

and $(z, s)$ be the solution of

$$
\left\{\begin{array}{l}
-\frac{\partial s}{\partial t}-\Delta s+s+\frac{\partial z}{\partial x_{1}}=\phi_{\alpha} 1_{\mathcal{O}} \quad \text { in } \Omega \times\left(0, T_{0}\right) \\
s+\Delta z=0 \quad \text { in } \Omega \times\left(0, T_{0}\right) \\
z=\frac{\partial z}{\partial n}=0 \quad \text { on } \Gamma \times\left(0, T_{0}\right) \\
s\left(T_{0}\right)=0 \quad \text { in } \Omega
\end{array}\right.
$$

Then $\left(z_{\alpha}, s_{\alpha}\right)=(z+\tilde{z}, s+\tilde{s})$. On the other hand, let $\left(\phi_{\alpha}, \theta_{\alpha}\right)$ the solution of

$$
\left\{\begin{array}{l}
\frac{\partial \theta_{\alpha}}{\partial t}-\Delta \theta_{\alpha}+\theta_{\alpha}-\frac{\partial \phi_{\alpha}}{\partial x_{1}}=0 \quad \text { in } \Omega \times\left(0, T_{0}\right) \\
\theta_{\alpha}+\Delta \phi_{\alpha}=0 \quad \text { in } \Omega \times\left(0, T_{0}\right) \\
\phi_{\alpha}=\frac{\partial \phi_{\alpha}}{\partial n}=0 \quad \text { on } \Gamma \times\left(0, T_{0}\right) \\
-\alpha \phi_{\alpha}(0)=z_{\alpha}(0) \quad \text { in } \Omega
\end{array}\right.
$$

where we have considered the control as $h_{\alpha}=\phi_{\alpha} 1_{\mathcal{O}}$. Problem (3.12) is reduced to the following:

Find $e \in \mathrm{H}_{0}^{1}(\Omega)$ such that

$$
(\alpha I+\Lambda) e=-\tilde{z}(0)
$$

\subsubsection{Time discretization}

Assuming that $T_{0}$ is finite, we introduce a discretization time step $\Delta t$, defined by $\Delta t=T_{0} / N$, where $N$ is a positive integer. Using an implicit Euler time discretization, we approximate (3.17) by $\theta^{0}=-\Delta e$.

Then assuming that $\left(\phi^{n-1}, \theta^{n-1}\right)$ is known, we solve for $n=1, \ldots, N$ :

$$
\left\{\begin{array}{l}
\frac{\left(\theta^{n}-\theta^{n-1}\right)}{\Delta t}-\Delta \theta^{n}+\theta^{n}-\frac{\partial \phi^{n}}{\partial x_{1}}=0 \quad \text { in } \Omega \\
\theta^{n}+\Delta \phi^{n}=0 \quad \text { in } \Omega \\
\phi^{n}=\frac{\partial \phi^{n}}{\partial n}=0 \quad \text { on } \Gamma
\end{array}\right.
$$

where $\theta^{n}=\theta(n \Delta t)$. For every $n$ this elliptic system has a unique solution (rewrite for example the system in terms of the variable $\phi^{n}$ ). For simplicity we have dropped the subscripts $\alpha$ in (3.17). Let us recall that instead of solving (3.3) we solve separately (3.15) and (3.16). We start (3.16) with $\left(z^{N+1}, s^{N+1}\right)=(0,0)$, and assuming that $\left(z^{n+1}, s^{n+1}\right)$ is known, we solve the following problem, for $n=N, N-1, \ldots, 1$, which also has a unique 
solution:

$$
\left\{\begin{array}{l}
\frac{\left(s^{n}-s^{n+1}\right)}{\Delta t}-\Delta s^{n}+s^{n}+\frac{\partial z^{n}}{\partial x_{1}}=\phi^{n} 1_{\mathcal{O}} \text { in } \Omega \\
s^{n}+\Delta z^{n}=0 \quad \text { in } \Omega \\
z^{n}=\frac{\partial z^{n}}{\partial n}=0 \quad \text { on } \Gamma .
\end{array}\right.
$$

We approximate $\Lambda$ by $\Lambda^{\Delta t}$, which is defined as

$$
\Lambda^{\Delta t} e=z^{1} .
$$

We can prove that the operator $\Lambda^{\Delta t}$ is symmetric and positive semi-definite. Indeed, for $e$ and $\tilde{e}$ in $\mathrm{H}_{0}^{1}(\Omega)$, we have

$$
\left(\Lambda^{\Delta t} e, \tilde{e}\right)_{1, \Omega}=\int_{\Omega} \nabla z^{1} \cdot \nabla \xi^{0} \mathrm{~d} x
$$

where, from $(3.14), \xi^{0}=\tilde{e}$.

Since $\Delta z^{N+1}=0$, it follows that

$$
\Delta z^{1} \xi^{0}=\Delta t \sum_{n=1}^{N}\left[\frac{\Delta z^{n}-\Delta z^{n+1}}{\Delta t} \xi^{n}-\frac{\xi^{n}-\xi^{n-1}}{\Delta t} \Delta z^{n}\right]
$$

Integrating (3.20) over $\Omega$, taking into account (3.19), and the approximation of system (3.14) in terms of $z$, we get after some integrations by parts

$$
\begin{aligned}
\left(\Lambda^{\Delta t} e, \tilde{e}\right)_{1, \Omega} & =-\int_{\Omega} \Delta z^{1} \xi^{0} \mathrm{~d} x=-\Delta t \sum_{n=1}^{N} \int_{\Omega}\left[\left(\Delta^{2} z^{n}-\Delta z^{n}+\frac{\partial z^{n}}{\partial x_{1}}\right) \xi^{n}-\frac{\xi^{n}-\xi^{n-1}}{\Delta t} \Delta z^{n}\right] \mathrm{d} x \\
& =-\Delta t \sum_{n=1}^{N} \int_{\Omega}\left[\left(\Delta^{2} \xi^{n}-\Delta \xi^{n}-\frac{\partial \xi^{n}}{\partial x_{1}}\right) z^{n}-\frac{\Delta \xi^{n}-\Delta \xi^{n-1}}{\Delta t} z^{n}\right] \mathrm{d} x=\Delta t \sum_{n=1}^{N} \int_{\mathcal{O}} \phi^{n} \xi^{n} \mathrm{~d} x .
\end{aligned}
$$

This shows that $\Lambda^{\Delta t}$ is symmetric and positive semi-definite.

For (3.15), we compute the approximate solution $(\tilde{z}, \tilde{s})$ by

$$
\tilde{z}^{N+1}=\varphi_{0}
$$

and for $n=1, \ldots, N$, assuming that $\left(\tilde{z}^{n+1}, \tilde{s}^{n+1}\right)$ is known, we solve the following system

$$
\left\{\begin{array}{l}
\frac{\left(\tilde{s}^{n}-\tilde{s}^{n+1}\right)}{\Delta t}-\Delta \tilde{s}^{n}+\tilde{s}^{n}+\frac{\partial \tilde{z}^{n}}{\partial x_{1}}=0 \quad \text { in } \Omega, \\
\tilde{s}^{n}+\Delta \tilde{z}^{n}=0 \quad \text { in } \Omega \\
\tilde{z}^{n}=\frac{\partial \tilde{z}^{n}}{\partial n}=0 \quad \text { on } \Gamma .
\end{array}\right.
$$

Finally, we approximate problem (3.18) by:

Find $\mathrm{e}^{\Delta t} \in \mathrm{H}_{0}^{1}(\Omega)$ such that

$$
\alpha\left(\mathrm{e}^{\Delta t}, v\right)_{1, \Omega}+\left(\Lambda^{\Delta t} \mathrm{e}^{\Delta t}, v\right)_{1, \Omega}=-\left(\tilde{z}^{1}, v\right)_{1, \Omega} \quad \forall v \in \mathrm{H}_{0}^{1}(\Omega) .
$$


Remark 3.2. The Euler schemes which have been used to discretize problem (3.12) in time are first order accurate. We can improve this by employing the Leap-frog scheme or the semi-Lagrangian scheme which have been used in [3].

\subsubsection{Space discretization}

We introduce $\left\{\mathcal{T}_{h}\right\}$, a regular family of triangulation of $\bar{\Omega}$, where $h=\max _{T \in \mathcal{T}_{h}} h_{T}$, with $h_{T}=\operatorname{diam}(T)$ $\forall T \in \mathcal{T}_{h}$. Next, we approximate $\mathrm{H}^{1}(\Omega)$ and $\mathrm{H}_{0}^{1}(\Omega)$ by the following finite dimensional spaces, with $\mathcal{P}_{1}(T)$ the space of polynomial functions of degree $\leq 1$,

$$
\mathcal{L}_{h}:=\left\{\mu_{h} \in \mathrm{H}^{1}(\Omega):\left.\mu_{h}\right|_{T} \in \mathcal{P}_{1}(T) \forall T \in \mathcal{T}_{h}\right\}
$$

and define

$$
\Phi_{h}:=\mathcal{L}_{h} \cap \mathrm{H}_{0}^{1}(\Omega)
$$

We approximate $\mathrm{L}^{2}(\Omega)$ by $\mathcal{L}_{h}$; this is reasonable since the closure of $\mathrm{H}^{1}(\Omega)$ in $\mathrm{L}^{2}(\Omega)$ is $\mathrm{L}^{2}(\Omega)$.

Problem (3.22) will be approximated by:

Find $e_{h}^{\Delta t} \in \Phi_{h}$ such that

$$
\alpha \int_{\Omega} \nabla e_{h}^{\Delta t} \cdot \nabla v_{h} \mathrm{~d} x+\int_{\Omega} \nabla\left(\Lambda_{h}^{\Delta t} e_{h}^{\Delta t}\right) \cdot \nabla v_{h} \mathrm{~d} x=-\int_{\Omega} \nabla \tilde{z}_{h}^{1} \cdot \nabla v_{h} \mathrm{~d} x \quad \forall v_{h} \in \Phi_{h},
$$

where $\Lambda_{h}^{\Delta t}$ and $\tilde{z}_{h}^{1}$ are obtained as described below.

The term $\tilde{z}_{h}^{1}$ is obtained from the following full discretization of (3.15):

$$
\tilde{z}_{h}^{N+1}=\varphi_{0 h} \quad \text { with } \varphi_{0 h} \in \Phi_{h} \text { being an approximation of } \varphi_{0}
$$

and for $n=N, N-1, \ldots, 1$, compute $\left(\tilde{z}_{h}^{n}, \tilde{s}_{h}^{n}\right) \in \Phi_{h} \times \mathcal{L}_{h}$ from $\left(\tilde{z}_{h}^{n+1}, \tilde{s}_{h}^{n+1}\right)$ by

$$
\begin{cases}\int_{\Omega} \frac{\left(\tilde{s}_{h}^{n}-\tilde{s}_{h}^{n+1}\right)}{\Delta t} v_{h} \mathrm{~d} x+\int_{\Omega} \overrightarrow{\operatorname{curl}} \tilde{s}_{h}^{n} \cdot \operatorname{curl} v_{h} \mathrm{~d} x+\int_{\Omega} \tilde{s}_{h}^{n} v_{h} \mathrm{~d} x+\int_{\Omega} \frac{\partial \tilde{z}_{h}^{n}}{\partial x_{1}} v_{h} \mathrm{~d} x=0 \\ \int_{\Omega} \tilde{s}_{h}^{n} \mu_{h} \mathrm{~d} x-\int_{\Omega} \operatorname{curl} \tilde{z}_{h}^{n} \cdot \operatorname{curl} \mu_{h} \mathrm{~d} x=0 & \forall \mu_{h} \in \mathcal{L}_{h},\end{cases}
$$

where $\int_{\Omega} \tilde{s}^{N+1} v_{h} \mathrm{~d} x=\int_{\Omega} \nabla \varphi_{0 h} \cdot \nabla v_{h} \mathrm{~d} x$.

The operator $\Lambda_{h}^{\Delta t}$ is defined by

$$
\Lambda_{h}^{\Delta t} e_{h}=z_{h}^{1} \quad \forall e_{h} \in \Phi_{h}
$$

where we solve the following discrete cascade systems to obtain $z_{h}^{1}$ from $e_{h}$ :

First problem. Given $\phi_{h}^{0}=e_{h}$, then for $n=1, \ldots, N$, we compute $\left(\phi_{h}^{n}, \theta_{h}^{n}\right) \in \Phi_{h} \times \mathcal{L}_{h}$ from $\left(\phi_{h}^{n-1}, \theta_{h}^{n-1}\right)$, the solution of

$$
\begin{cases}\int_{\Omega} \frac{\left(\theta_{h}^{n}-\theta_{h}^{n-1}\right)}{\Delta t} v_{h} \mathrm{~d} x+\int_{\Omega} \operatorname{curl} \theta_{h}^{n} \cdot \operatorname{curl} v_{h} \mathrm{~d} x+\int_{\Omega} \theta_{h}^{n} v_{h} \mathrm{~d} x-\int_{\Omega} \frac{\partial \phi_{h}^{n}}{\partial x_{1}} v_{h} \mathrm{~d} x=0 \\ \forall v_{h} \in \Phi_{h}, \\ \int_{\Omega} \theta_{h}^{n} \mu_{h} \mathrm{~d} x-\int_{\Omega} \overrightarrow{\operatorname{curl}} \phi_{h}^{n} \cdot \operatorname{curl} \mu_{h} \mathrm{~d} x=0 & \forall \mu_{h} \in \mathcal{L}_{h},\end{cases}
$$

where $\int_{\Omega} \theta_{h}^{0} v_{h} \mathrm{~d} x=\int_{\Omega} \nabla \phi_{h}^{0} \cdot \nabla v_{h} \mathrm{~d} x$. 
Second problem. Given $s_{h}^{N+1}=0$, then for $n=N, N-1, \ldots, 1$, we compute $\left(z_{h}^{n}, s_{h}^{n}\right) \in \Phi_{h} \times \mathcal{L}_{h}$ from $\left(z_{h}^{n+1}, s_{h}^{n+1}\right)$, the solution of

$$
\left\{\begin{array}{l}
\int_{\Omega} \frac{\left(s_{h}^{n}-s_{h}^{n+1}\right)}{\Delta t} v_{h} \mathrm{~d} x+\int_{\Omega} \overrightarrow{\operatorname{curl}} s_{h}^{n} \cdot \operatorname{curl} v_{h} \mathrm{~d} x+\int_{\Omega} s_{h}^{n} v_{h} \mathrm{~d} x+\int_{\Omega} \frac{\partial z_{h}^{n}}{\partial x_{1}} v_{h} \mathrm{~d} x=\int_{\mathcal{O}} \phi_{h}^{n} v_{h} \mathrm{~d} x \quad \forall v_{h} \in \Phi_{h}, \\
\int_{\Omega} s_{h}^{n} \mu_{h} \mathrm{~d} x-\int_{\Omega} \overrightarrow{\operatorname{curl}} z_{h}^{n} \cdot \operatorname{curl} \mu_{h} \mathrm{~d} x=0 \quad \forall \mu_{h} \in \mathcal{L}_{h},
\end{array}\right.
$$

with $\phi_{h}^{n}$ being the output of the first problem.

The discretization of time-dependent Navier-Stokes equations in the stream function and vorticity formulation has been studied by Bernardi et al. [4] and by Girault and Raviart [15] in the stationary case. We refer to [4], for studying uniqueness of the discrete solution for the above problems.

We can prove an analogous relation to (3.21) for the operator $\Lambda_{h}^{\Delta t}$ which shows that the operator is symmetric and positive semi-definite, implying that (3.23) has a unique solution.

To solve problem (3.23) we can use either direct methods or iterative methods (such as conjugate gradient). The conjugate gradient method has been employed in [7] to solve exact and approximate boundary controllability problems for the heat equation. In this work, we will use direct methods. When the dimension of the discrete domain is not too large, we can compute an explicit representation of $\Lambda_{h}^{\Delta t}$ in (3.23).

Let us denote by $\bar{x}_{1}, \ldots, \bar{x}_{k}$ the nodes of the triangulation. The first ones, $\bar{x}_{1}, \ldots, \bar{x}_{m}$ corresponding to the interior nodes and the last ones $\bar{x}_{m+1}, \ldots, \bar{x}_{k}$ to the nodes on the boundary. Then, any element of $\mathcal{L}_{h}$ may be expressed as

$$
\mu_{h}=\sum_{j=1}^{k} \mu_{j} \varphi_{j}(x) \quad \text { with } \mu_{j}=\mu_{h}\left(\bar{x}_{j}\right)
$$

and any element of $\Phi_{h}$ may be written as

$$
v_{h}=\sum_{j=1}^{m} v_{j} \varphi_{j}(x) \quad \text { with } v_{j}=v_{h}\left(\bar{x}_{j}\right)
$$

where $\varphi_{1}, \ldots, \varphi_{k}$ are the standard basis functions for these elements, satisfying $\varphi_{i}\left(\bar{x}_{l}\right)=\delta_{i l}$. Thus, problem (3.23) is reduced to:

Find $e_{1}^{\Delta t}, \ldots, e_{m}^{\Delta t}$ such that

$$
\begin{array}{r}
\sum_{j=1}^{m}\left(\alpha \int_{\Omega} \nabla \varphi_{j} \cdot \nabla \varphi_{i} \mathrm{~d} x+\int_{\Omega} \nabla\left(\Lambda_{h}^{\Delta t} \varphi_{j}\right) \cdot \nabla \varphi_{i} \mathrm{~d} x\right) e_{j}^{\Delta t}=-\sum_{j=1}^{m} \tilde{z}_{j}^{1} \int_{\Omega} \nabla \varphi_{j} \cdot \nabla \varphi_{i} \mathrm{~d} x \\
i=1, \ldots, m .
\end{array}
$$

This can be written in matrix form as follows:

$$
(\alpha I+A) E^{\Delta t}=-Z^{1}
$$

where, $E^{\Delta t}=\left(e_{1}^{\Delta t}, \ldots, e_{m}^{\Delta t}\right)$ is the unknown vector and $Z^{1}=\left(\tilde{z}_{1}^{1}, \ldots, \tilde{z}_{m}^{1}\right)$ is the vector solution of (3.24) associated with $\varphi_{0 h}$. To obtain each column of the matrix $A=\left(a_{i j}\right), i, j=1, \ldots, m$, we solve (3.25)-(3.26) for each basis function $\varphi_{j}, j=1, \ldots, m$, as the initial condition $e_{h}$ for (3.25). Then, the vector of coefficient of the final result $z_{h}^{1}$ in the basis $\left\{\varphi_{j}\right\}_{j=1}^{m}$ gives the $j$ th column of matrix $A$.

If the solution of (3.23) for each $\varphi_{0 h}$ is attained at $e_{h}^{\Delta t}$, we compute (3.25) with the initial condition $\phi_{h}^{0}=e_{h}^{\Delta t}$. Then, we obtain the optimal control as $\hat{h}_{h}^{n}=\hat{\phi}_{h}^{n} 1_{\mathcal{O}}$ and the associated solution of $(3.26)$ by $\left(\hat{z}_{h}^{n}, \hat{s}_{h}^{n}\right)$. 
To compute an approximation of $\psi\left(T_{0}\right)$, denoted by $\psi_{\text {rec, } h}$, we must choose a suitable discrete basis (of small dimension) $\left(\varphi_{0}^{j}\right)$ and their corresponding finite element approximation $\varphi_{0 h}^{j}$. Notice that, we should compute (3.27) for each element of $\varphi_{0 h}^{j}$. A natural selection should be the canonical finite element basis $\varphi_{j}$, but, as we will see later on in the numerical examples, this choice is quite expensive in terms of computer time, since in this case (3.27) must be solved as many times as the number of degrees of freedom $m$ of the mesh. Instead, in order to minimize the computer time, we propose to make the eigenvalue decomposition of the Laplacian and take the eigenvectors basis, namely $\left\{u_{k}\right\}_{k=1, \ldots, l}$, where $l$ is the number of eigenvalues considered. In fact, the computational cost is dramatically reduced as we will see later on in the numerical tests. From (3.6) we have the following approximation (when $\alpha \rightarrow 0$ )

$$
\int_{\Omega} \nabla \psi_{\mathrm{rec}, h} \cdot \nabla u_{k} \mathrm{~d} x=\Delta t \sum_{n=1}^{N} \int_{\Omega} \mathcal{T}_{h}^{n} \cdot \operatorname{curl}\left(\hat{z}_{h}^{n}\left(u_{k}\right)+\tilde{z}_{h}^{n}\left(u_{k}\right)\right) \mathrm{d} x-\Delta t \sum_{n=1}^{N} \int_{\mathcal{O}} \psi_{\mathrm{obs}, h}^{n} \hat{\phi}_{h}^{n}\left(u_{k}\right) \mathrm{d} x \quad k=1, \ldots, l,
$$

where $\left(\mathcal{T}_{h}^{n}\right)$ and $\left(\psi_{\mathrm{obs}, h}^{n}\right)$ are the wind stress and the measurements of the stream function in $\mathcal{O} \times\left(0, T_{0}\right)$, respectively.

To compute $\psi_{\text {rec }, h}$ we proceed as follows. Since

$$
\begin{aligned}
\psi_{\mathrm{rec}, h} & =\sum_{i=1}^{m} \Psi_{i}^{N} \varphi_{i}(x) \quad \text { with } \Psi_{i}^{N}=\psi_{\mathrm{rec}, h}\left(\bar{x}_{i}\right), \\
\varphi_{j} & =\sum_{k=1}^{l}\left(u_{k}, \varphi_{j}\right) u_{k},
\end{aligned}
$$

we have

$$
\begin{aligned}
\int_{\Omega} \nabla \psi_{\mathrm{rec}, h} \cdot \nabla \varphi_{j} \mathrm{~d} x & =\sum_{i=1}^{m} \int_{\Omega} \Psi_{i}^{N} \nabla \varphi_{i} \cdot \nabla \varphi_{j} \mathrm{~d} x \quad j=1, \ldots, m \\
& =\sum_{k=1}^{l} \int_{\Omega}\left(u_{k}, \varphi_{j}\right) \nabla \psi_{\mathrm{rec}, h} \cdot \nabla u_{k} \mathrm{~d} x
\end{aligned}
$$

If we introduce $K_{i j}=\int_{\Omega} \nabla \varphi_{i} \cdot \nabla \varphi_{j} \mathrm{~d} x$, with $i=1, \ldots, m$ and $j=1, \ldots, m$, it follows that

$$
\hat{\Psi}^{N}=K^{-1} \sum_{j=1}^{m} \sum_{k=1}^{l}\left(u_{k}, \varphi_{j}\right) \int_{\Omega} \nabla \psi_{\mathrm{rec}, h} \cdot \nabla u_{k} \mathrm{~d} x
$$

where $\hat{\Psi}^{N}=\left(\Psi_{1}^{N}, \ldots, \Psi_{m}^{N}\right)$ is the vector that we are looking for.

Table 1 summarizes the implementation of the discrete method and shows the dependence of each stage on the principal parameters of the problem.

\section{NumERICAL EXPERIMENTS}

In this section, we present several numerical experiments. Let $\Omega=[0,1] \times[0,1]$ and $T_{0}=0.05$. For the Rossby, Munk and Stommel numbers, we consider (taken from [3]):

$$
R_{o}=1.5 \times 10^{-3}, \quad \epsilon_{m}=1 \times 10^{-4}, \quad \epsilon_{s}=5 \times 10^{-3},
$$


TABLE 1. Summary of the numerical implementation. NV denotes the number of nodes, NE the number of eigenvalues and NT the number of time intervals.

\begin{tabular}{|c|c|c|c|}
\hline & Stage & Applied method & Dependence on \\
\hline 1 & $\begin{array}{c}\text { Mesh and } \\
\text { elementary matrices }\end{array}$ & $\mathcal{P}_{1}$-finite elements & $\Omega$ \\
\hline 2 & Computation of $\Lambda$ & $\begin{array}{c}\text { Evolution linear system }(3.25)-(3.26) \\
\text { of } \mathrm{NT}(2 \mathrm{NV} \times 2 \mathrm{NV}) \text { for each } \varphi_{i} \\
i=1, \ldots, \mathrm{NV} \\
+ \text { NT matrix vector products }\end{array}$ & $\varphi_{i}, \mathcal{O}$ \\
\hline 3 & $\begin{array}{c}\text { Right - hand side (RHS) } \\
\text { of }(3.23)\end{array}$ & $\begin{array}{c}\text { Evolution linear system }(3.24) \\
\text { of } \mathrm{NT}(2 \mathrm{NV} \times 2 \mathrm{NV}) \text { for each } u_{k}, \\
k=1, \ldots, \mathrm{NE}\end{array}$ & $u_{k}$ \\
\hline 4 & Optimality system (3.23) & $\begin{array}{c}\text { Simultaneous linear system } \\
\text { of } \mathrm{NV} \times \mathrm{NV} \text { for each } \mathrm{RHS} \\
k=1, \ldots, \mathrm{NE}\end{array}$ & $u_{k}, \mathcal{O}, \alpha$ \\
\hline 5 & $\begin{array}{c}\text { Projection }\left(\nabla \psi_{\text {rec }, h}, \nabla u_{k}\right) \\
(3.28)\end{array}$ & $\begin{array}{c}\text { Linear combination } \\
\text { of steps } 2,3 \text { and } 4 \text {, and } \\
\text { matrix vector products }\end{array}$ & $\mathcal{T}, \psi_{\text {obs }}$ \\
\hline 6 & Prediction & $\begin{array}{c}\text { Evolution linear system }(1.1) \\
\mathrm{NT}(2 \mathrm{NV} \times 2 \mathrm{NV})\end{array}$ & $\mathcal{T}, \psi_{\text {rec }, h}$ \\
\hline
\end{tabular}

which correspond to

$$
\begin{aligned}
& \gamma=1 \times 10^{-7} \mathrm{~s}^{-1}, \quad A_{H}=2 \times 10^{3} \mathrm{~m}^{2} \cdot \mathrm{s}^{-1}, \quad L=10^{6} \mathrm{~m}, \quad T=1 \text { year } \\
& U=0.03 \mathrm{~m} \cdot \mathrm{s}^{-1}, \quad \beta=2 \times 10^{-11} \mathrm{~m}^{-1} \cdot \mathrm{s}^{-1}, \quad D_{0}=800 \mathrm{~m} .
\end{aligned}
$$

For wind stress, we use

$$
\mathcal{T}=\left(\tau_{1}, \tau_{2}\right)=10^{-2} \exp \left(\pi^{2} t\right)\left(-\frac{1}{\pi} \cos \left(\pi \frac{x_{2}}{L}\right), 0\right)
$$

The following series of test problems have been done with mesh size $h=1 / 40$ and time step size $\Delta t=T_{0} / 50$. As we have no real measurements for testing our method, we will compare the results of our experiments with the results of the original model (1.1), i.e., we compute the ocean circulation using (1.1) over the time interval $\left(0, T_{0}\right)$, for initial given value $\Delta \psi(0)=-\sin \left(\pi x_{1}\right) \sin \left(\pi x_{2}\right)$ and surface wind stress (4.1). Then, we save $\psi_{h}$ in the observatory $\mathcal{O} \times\left(0, T_{0}\right)$ and $\psi_{h}^{N}$, which will be our exact target values. In Figure 1, we show the evolution of the stream function for different interval of time.

We compute $\int_{\Omega} \nabla \psi_{\text {rec }, h} \cdot \nabla u_{k} \mathrm{~d} x, k=1, \ldots, l$, following the algorithm presented in Table 1 . In the experiments bellow, we compute a relative error given by:

$$
\text { Error }=\frac{\left\|\psi_{h}^{N}-\psi_{\mathrm{rec}, h}\right\|_{0, \Omega}}{\left\|\psi_{h}^{N}\right\|_{0, \Omega}}
$$

First, let us choose the penalty parameter $\alpha$. To do this, we present, in Figure 2, the graph of the two terms of the functional (3.1) for different values of $\alpha$, i.e., the control norm $\|h\|_{\mathrm{L}^{2}\left(\mathrm{~L}^{2}(\mathcal{O})\right)}^{2}$ and $|z(0)|_{1, \Omega}^{2}$. Here, we consider the observation set $\mathcal{O}$ as $[0,1] \times[0.3,0.7]$ and 60 eigenvalues. We see that the term $\|h\|_{\mathrm{L}^{2}\left(\mathrm{~L}^{2}(\mathcal{O})\right)}^{2}$ 

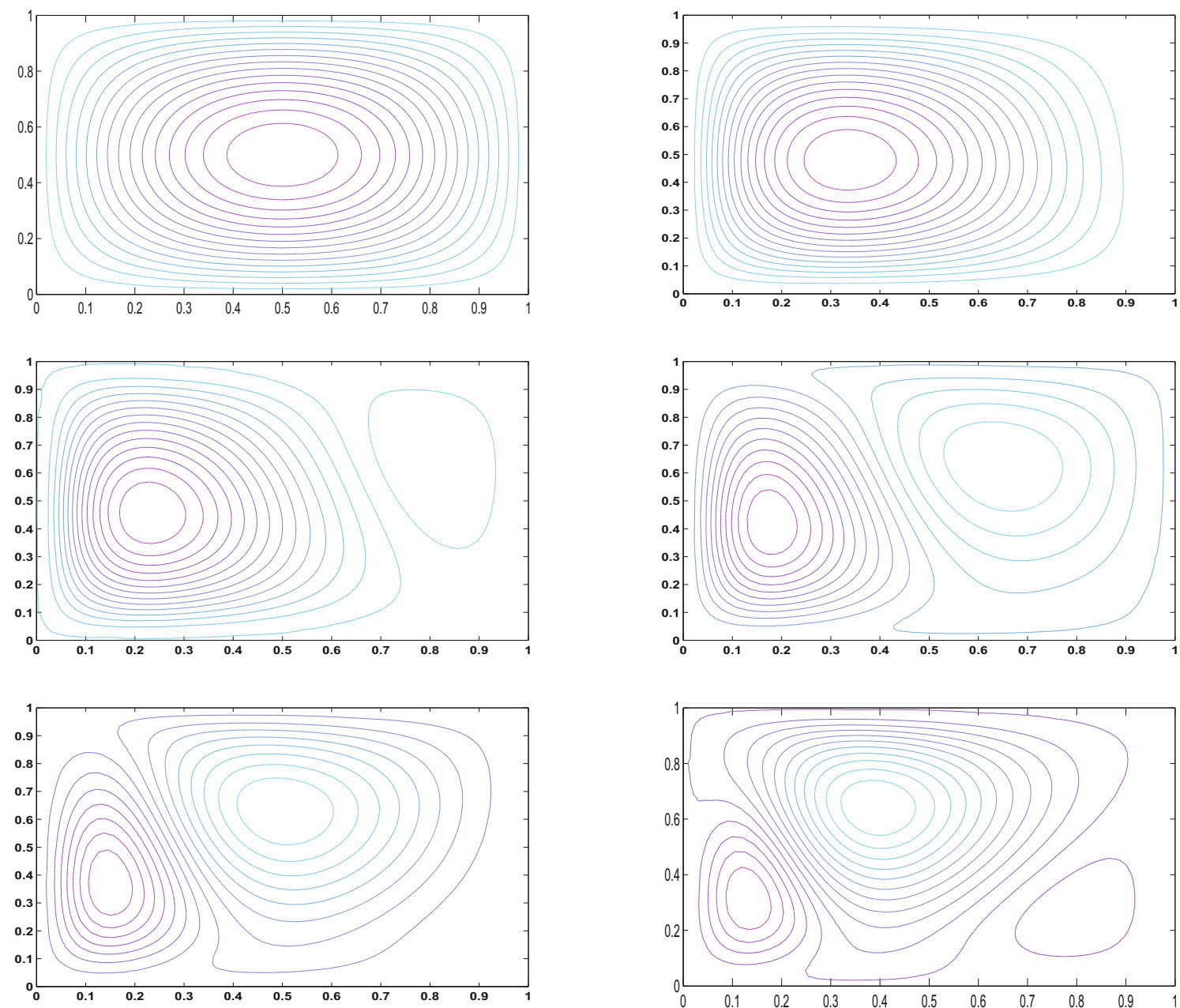

FiguRE 1. Evolution of the exact stream function in time.

tends to increase and the term $|z(0)|_{1, \Omega}^{2}$ tends to zero as $\alpha$ tends to zero. We have chosen $\alpha=0.025$ which corresponds to the value of maximum curvature in the graphic, which is the classical L-curve criterion [16].

For the following experiments, we assume that the observation data $\left(\psi_{\mathrm{obs}, h}^{n}\right)$ have certain observation error of random distribution:

$$
\hat{\psi}_{\mathrm{obs}, h}^{n}=\psi_{\mathrm{obs}, h}^{n}\left(1+\delta R\left(x_{1}, x_{2}, t\right)\right),
$$

where $R\left(x_{1}, x_{2}, t\right)$ denotes a random function varying in the range $[-1,1]$, and $\delta$ is the parameter representing the noise level.

As mentioned in the previous section, the computational cost of our approach depends linearly on the size of the discrete basis function $\varphi_{0 h}$ chosen. Figure 3 shows that, in order to obtain a recovery error similar to the error using the standard finite element basis (2545 degrees of freedom), it is sufficient to consider the first 60 eigenvalues of the Laplacian eigenvalue decomposition (60 degrees of freedom) computed in the same mesh. This means that, for the same accuracy, the cost of the data assimilation method is reduced more that 40 times when using a spectral basis. This number of eigenvalues (60) is also near the sampling limit compatible with the resolution of the mesh. 


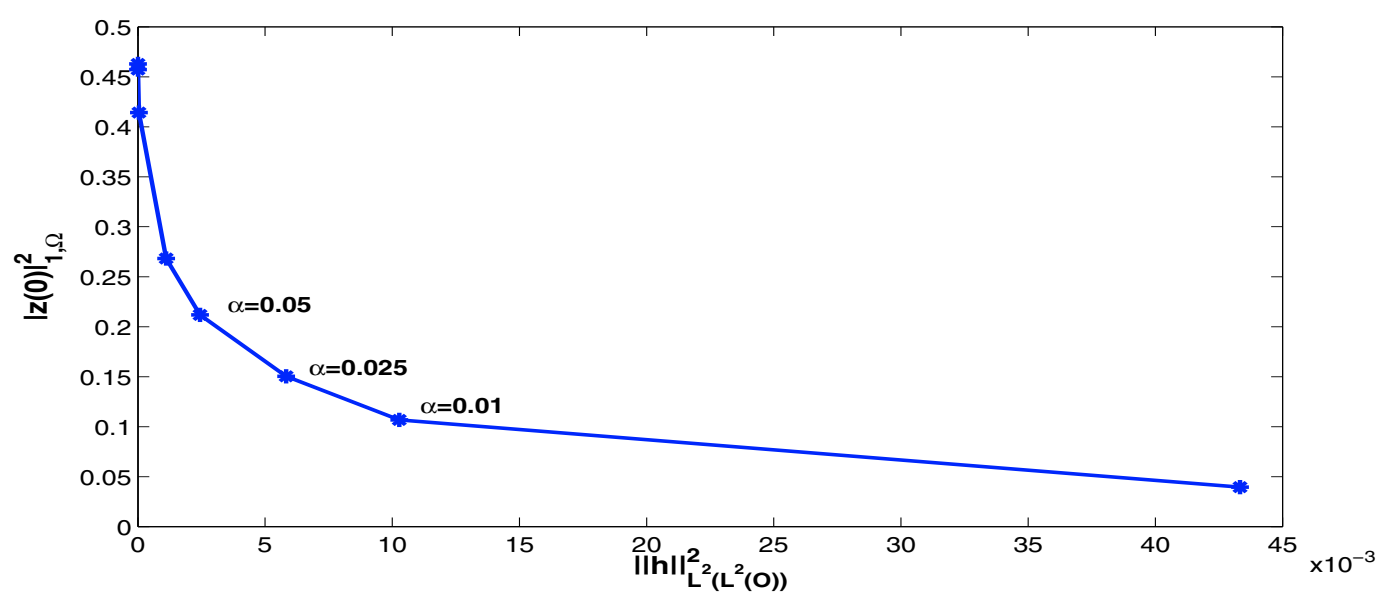

Figure 2. Control norm $\|h\|_{\mathrm{L}^{2}\left(\mathrm{~L}^{2}(\mathcal{O})\right)}^{2}$ versus the term $|z(0)|_{1, \Omega}^{2}$ for different values of the penalty parameter $\alpha$. The observatory $\mathcal{O}$ for this test is $[0,1] \times[0.3,0.7]$. We have chosen for our tests $\alpha=0.025$.

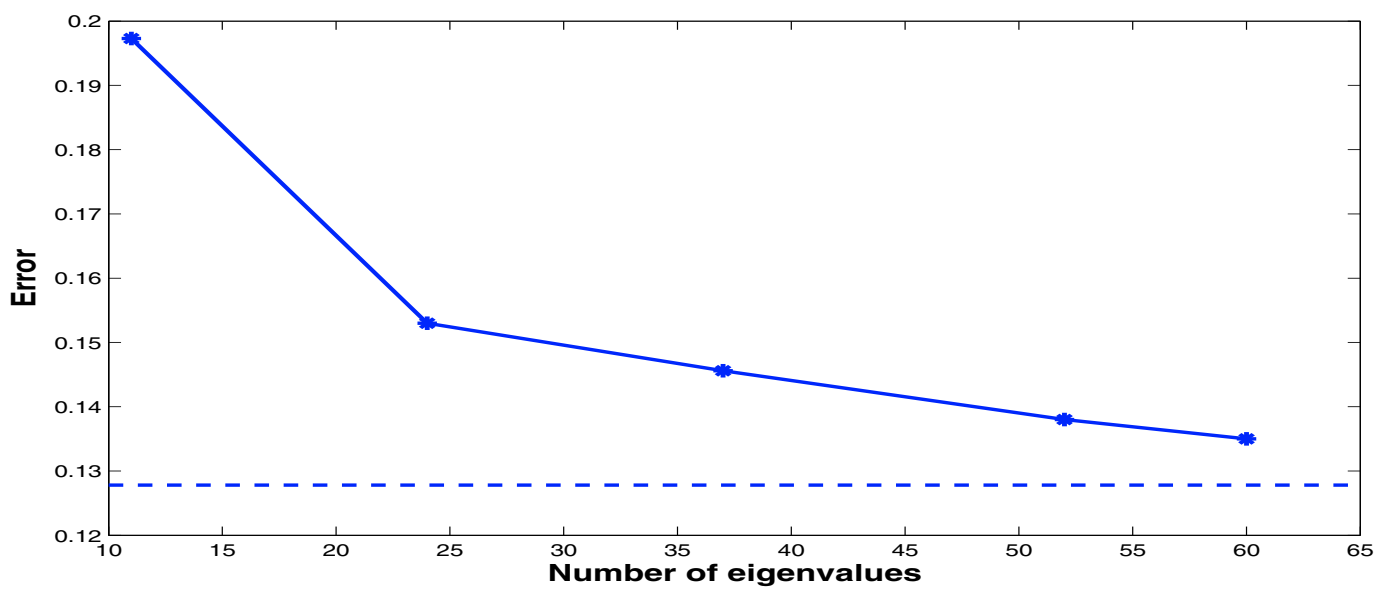

FiguRE 3. Relative error versus the number of eigenvalues taken for the projection in the assimilation method (bold line). The error level obtained using a canonical finite element basis for the same mesh is indicated by a horizontal dashed line. The observatory $\mathcal{O}$ for this test is $[0,1] \times[0.3,0.7]$. We have chosen 60 eigenvalues for our tests. The regularizing parameter is $\alpha=0.025$ and the noise level $\delta=1 \%$.

In order to simulate better the reality of the measurements, four observatories in the form of circles were considered initially. The location of them was random within the domain, centering in $(0.2,0.4),(0.2,0.6)$, $(0.3,0.2),(0.3,0.8)$ with radio equal to 0.1 . Figure 4 shows the distribution of the observatories, numerical reconstruction of stream function, and relative percentage error between recovered stream function and exact solution at $T_{0}$.

Using the information of Figure 4, we added two observatories in the zones where we obtain more differences $((0.1,0.2),(0.5,0.5))$. In Figure 5 we can see how the errors fell considerably.

In Table 2, we present the relative errors in $\mathrm{L}^{2}(\Omega)$ and $\mathrm{H}^{1}(\Omega)$ for the final recovered stream function in both cases, with and without noise in the observatory set, using 60 eigenvalues. Notice that, increasing the number 

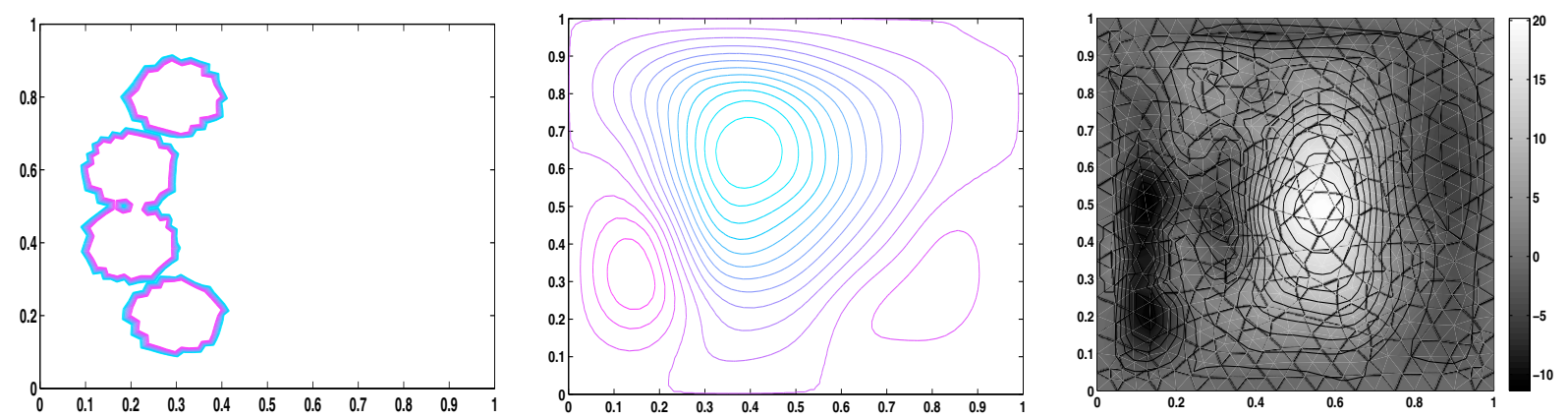

FIGURE 4. Left: Location of the observatories. Center: Recovered stream function at $T_{0}$ using four observatories. Right: Relative percentage error between recovered stream function and exact solution at $T_{0}$. The regularizing parameter is $\alpha=0.025$ and the noise level $\delta=0$.
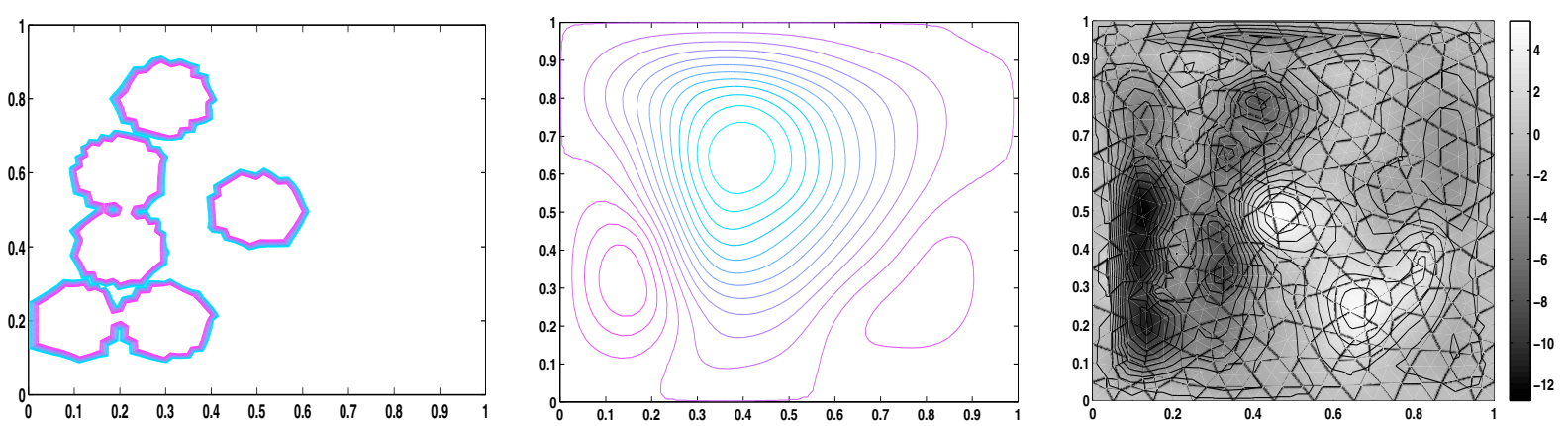

Figure 5. Left: Location of the observatories. Center: Recovered stream function at $T_{0}$ using six observatories. Right: Relative percentage error between recovered stream function and exact solution at $T_{0}$. Notice the change of grey scale with respect to Figure 4 . The regularizing parameter is $\alpha=0.025$ and the noise level $\delta=0$.

of observatories we can increase the noise level in the measurements maintaining a satisfactory reconstruction of the stream function at $T_{0}$.

\section{Comments And CONCLUSiOns}

A non classical approach to data assimilation based on exact controllability is proposed. More precisely, the problem is to recover the final state value $\psi\left(T_{0}\right)$ of a quasi-geostrophic ocean model in order to predict the future state in the time interval $\left(T_{0}, T\right)$. The inverse initial value problem is ill-posed so the value at $t=T_{0}$, which plays the role of initial value on the interval $\left(T_{0}, T\right)$ is really computed as the final condition of the previous interval $\left(0, T_{0}\right)$ without knowledge of the initial value at time $t=0$. In Section 1 , we stated the model and the data assimilation problem. In Section 2, the null controllability and the exact reconstruction of the state at $T_{0}$ was proved by means of an observability inequality derived from a global Carleman estimate for the associated velocity-pressure formulation.

In Section 3, we presented an approximate algorithm which makes use of classical optimal control techniques and regularization. In Theorem 3.1, we proved the convergence of the regularizing solutions.

In all the experiments presented in Section 4, we used a reduced basis approach in order to decrease the computational cost of the proposed data assimilation method. We studied the role played by the observatory size for the recovery of the final value at $T_{0}$. The numerical results presented in Section 4 show that our approach behaves well in the presence of noise in the observed measurements. 
TABLE 2. Relative errors in $L^{2}(\Omega)$ and $H^{1}(\Omega)$ for the final recovered stream function with noise in the measurements and in the absence of noise $(\delta=0)$ versus the observatory size $\mathcal{O}$.

\begin{tabular}{|c|c|c|c|}
\hline Number of $\mathcal{O}$ & Noise level $\delta$ & $\frac{\left\|\psi_{h}^{N}-\psi_{\text {rec, },}\right\|_{0, \Omega}}{\left\|\psi_{h}^{N}\right\|_{0, \Omega}}$ & $\frac{\left|\psi_{h}^{N}-\psi_{\text {rec }, h}\right|_{1, \Omega}}{\left|\psi_{h}^{N}\right|_{1, \Omega}}$ \\
\hline 4 & 0 & 0.1813 & 0.2776 \\
\hline 4 & 0.01 & 0.1852 & 0.2786 \\
\hline 4 & 0.05 & 0.2034 & 0.2850 \\
\hline 4 & 0.1 & 0.2314 & 0.3018 \\
\hline 6 & 0 & 0.1007 & 0.2130 \\
\hline 6 & 0.08 & 0.1045 & 0.2232 \\
\hline 6 & 0.1 & 0.1154 & 0.2295 \\
\hline 6 & 0.15 & 0.1357 & 0.2440 \\
\hline
\end{tabular}

In this paper we have proposed an alternative data assimilation method applied to a simplified ocean model. The implementation to more realistic ocean or atmospheric models should be possible by using the same methodology presented here, but this is out of the scope of this study.

\section{REFERENCES}

[1] A. Belmiloudi and F. Brossier, A control method for assimilation of surface data in a linearized Navier-Stokes-type problem related to oceanography. SIAM J. Control Optim. 35 (1997) 2183-2197.

[2] A.F. Bennett, Inverse Methods in Physical Oceanography. Cambridge University Press, Cambridge (1992).

[3] R. Bermejo and P. Galán del Sastre, Numerical studies of the long-term dynamics of the 2D Navier-Stokes equations applied to ocean circulation, in XVII CEDYA: Congress on Differential Equations and Applications, L. Ferragut and A. Santos Eds., Universidad de Salamanca, Salamanca (2001) 15-34.

[4] C. Bernardi, E. Godlewski and G. Raugel, A mixed method for time-dependent Navier-Stokes problem. IMA J. Numer. Anal. 7 (1987) 165-189.

[5] E. Blayo, J. Blum and J. Verron, Assimilation variationnelle de données en océanographie et réduction de la dimension de l'espace de contrôle, in Équations aux dérivées partielles et applications, Articles dédiés à Jacques-Louis Lions, Gauthier-Villars, éd. Sci. Méd. Elsevier, Paris (1998) 199-219.

[6] J. Blum, B. Luong and J. Verron, Variational assimilation of altimeter data into a non-linear ocean model: Temporal strategies. ESAIM: Proc. 4 (1998) 21-57.

[7] C. Carthel, R. Glowinski and J.L. Lions, On exact and approximate boundary controllabilities for heat equation: a numerical approach. J. Optim. Theory Appl. 82 (1994) 429-484.

[8] P. Courtier, O. Talagrand, Variational assimilation of meteorological observations with the adjoint vorticity equation. I: Theory. Quart. J. Roy. Meteorol. Soc. 113 (1987) 1311-1328.

[9] C. Fabre, J.-P. Puel and E. Zuazua, Approximate controllability of the semilinear heat equation. Proc. Roy. Soc. Edinburgh Sect. A 125 (1995) 31-61.

[10] E. Fernández-Cara, S. Guerrero, O.Y. Imanuvilov and J.-P. Puel, Local exact controllability of the Navier-Stokes system. J. Math. Pures Appl. 83 (2004) 1501-1542.

[11] E. Fernández-Cara, G.C. García and A. Osses, Controls insensitizing the observation of a quasi-geostrophic ocean model. SIAM J. Control Optim. 43 (2005) 1616-1639.

[12] A.V. Fursikov and O.Y. Imanuilov, Local exact controllability of the two-dimensional Navier-Stokes equations. Matematicheskiı Sbornik 187 (1996) 103-138.

[13] A. Fursikov and O.Y. Imanuvilov, Controllability of evolution equations. Lecture Notes, Research Institute of Mathematics, Seoul National University, Korea (1996).

[14] M. Ghil and P. Malanotte-Rizzoli, Data assimilation in meteorology and oceanography. Adv. Geophys. 33 (1991) $141-266$.

[15] V. Girault and P.-A. Raviart, Finite Element Approximation of the Navier-Stokes Equations. Springer-Verlag, New York (1986).

[16] C. Hansen, Analysis of ill-posed problems by means of the L-curve. SIAM Rev. 34 (1992) 561-580. 
[17] F.-X. Le Dimet and O. Talagrand, Variational algorithms for analysis and assimilation of meteorological observations. Tellus 38A (1986) $97-110$.

[18] J.-L. Lions, Optimal Control of Systems Governed by Partial Differential Equations. Springer-Verlag, Berlin (1971).

[19] J.-L. Lions, Remarks on approximate controllability, Festschrift on the occasion of the 70th birthday of Samuel Agmon. J. Anal. Math. 59 (1992) 103-116.

[20] J.-L. Lions, Exact and approximate controllability for distributed parameter system, in VI Escuela de Otoño Hispano-Francesa sobre simulación numérica en física e ingeniería, Universidad de Sevilla, España (1994) 1-238.

[21] J.-L. Lions and E. Magenes, Problèmes aux limites non homogènes et applications 1. Dunod (1968).

[22] B. Luong, J. Blum and J. Verron, A variational method for the resolution of a data assimilation problem in oceanography. Inv. Probl. 14 (1998) 979-997.

[23] G.I. Marchuk, Formulation of theory of perturbations for complicated models. Appl. Math. Optim. 2 (1975) 1-33.

[24] P.G. Myers and A.J. Weaver, A diagnostic barotropic finite-element ocean circulation model. J. Atmos. Ocean Tech. 12 (1995) $511-526$.

[25] A. Osses and J.-P. Puel, Boundary controllability of a stationary Stokes system with linear convection observed on an interior curve. J. Optim. Theory Appl. 99 (1998) 201-234.

[26] A. Osses and J.-P. Puel, On the controllability of the Laplace equation observed on an interior curve. Rev. Mat. Complut. 11 (1998) 403-441.

[27] J.-P. Puel, Une approche non classique d'un problème d'assimilation de données. C. R. Math. Acad. Sci. Paris 335 (2002) $161-166$.

[28] J.-P. Puel, A nonstandard approach to a data assimilation problem and Tychonov regularization revisited. SIAM J. Control Optim. 48 (2009) 1089-1111.

[29] L. Quartapelle, Numerical Solution of the Incompressible Navier-Stokes Equations. Birkhauser Verlag (1993).

[30] J. Verron, Altimeter data assimilation into ocean model: sensitivity to orbital parameters. J. Geophys. Res. 95 (1990) 1144311459.

[31] J. Verron, Nudging satellite altimeter data into quasi-geostrophic ocean models. J. Geophys. Res. 97 (1992) 7479-7492. 